J. Phys. Chem. B, 2010, 114 (9), pp 3152-3169 ; DOI: 10.1021/jp911445m

\title{
Simultaneous control of emission localization and two-photon absorption efficiency in dissymmetrical chromophores
}

Claudine Katan, ${ }^{\dagger, \ddagger}$ Marina Charlot, ${ }^{\dagger, \ddagger}$ Olivier Mongin, ${ }^{\dagger, \ddagger}$ Céline Le Droumaguet, $^{\dagger, \ddagger}$ Viatcheslav Jouikov, ${ }^{\dagger, \ddagger}$ Francesca Terenziani, ${ }^{\S}$ Ekaterina Badaeva, ${ }^{\#, e}$ Sergei Tretiak,,$"$ and Mireille Blanchard-Desce $*,+$,

†, CNRS, Chimie et Photonique Moléculaires (UMR 6510), 263 avenue du Général Leclerc, 35042 Rennes, France,

*Université de Rennes 1, UMR 6510, Campus de Beaulieu, Bâtiment 10A, Case 1003, 35042 Rennes, France,

$\S$ Dipartimento di Chimica GIAF, Università di Parma \& INSTM-UdR Parma, Parco Area delle Scienze 17/a, 43100 Parma, Italy,

\# Department of Chemistry, University of Washington, Seattle, WA 98195-1700, USA,

" Los Alamos National Laboratory, Theoretical Division, Center for Nonlinear Studies (CNLS), and Center for Integrated Nanotechnologies (CINT), Los Alamos, New Mexico 87545, USA

* To whom correspondence should be addressed. E-mail: mireille.blancharddesce@univ-rennes1.fr (M.B.-D.); serg@lanl.gov (S. T.). 
J. Phys. Chem. B, 2010, 114 (9), pp 3152-3169 ; DOI: 10.1021/jp911445m

\begin{abstract}
:
The aim of the present work is to demonstrate that combined spatial tuning of fluorescence and two-photon absorption (TPA) properties of multipolar chromophores can be achieved by introduction of slight electronic chemical dissymmetry. In that perspective, two model series of structurally related chromophores have been designed and investigated. One is based on rod-like quadrupolar chromophores bearing either two identical or different electron-donating (D) end groups and the other on three-branched octupolar chromophores built from a trigonal donating moiety bearing identical or different acceptor (A) peripheral groups. The influence of the electronic dissymmetry is investigated by combined experimental and theoretical studies of the linear and nonlinear optical properties of dissymmetrical chromophores compared to their symmetrical counterparts. In both types of systems (i.e., quadrupoles and octupoles) experiments and theory reveal that excitation is essentially delocalized and that excitation involves synchronized charge redistribution (i.e., concerted intramolecular charge transfer) between the different D and A moieties within the multipolar structure. In contrast, the emission stems only from a particular dipolar subunit bearing the strongest D or A moiety due to fast excitation localization after excitation, prior to emission. Hence control of emission characteristics (polarization and emission spectrum), can be achieved - in addition to localization - by controlled introduction of electronic dissymmetry (i.e., replacement of one of the D or A end-groups by a slightly stronger D' or A' unit). Interestingly slight dissymmetrical functionalization of both quadrupolar and octupolar compounds does not lead to significant loss in TPA responses and can even be beneficial due to the spectral broadening and peak position tuning that it allows. This study thus reveals an original molecular engineering route allowing TPA enhancement in multipolar structures, due to concerted core-to-periphery or periphery-to-core intramolecular charge redistribution upon excitation, while providing for control of emission localization. Such a route could be extended to more intricate (dendritic) and multipolar (3D) systems.
\end{abstract}

\title{
Keywords:
}

nonlinear optics; multiphoton processes; fluorescence; symmetry-breaking; theoretical modeling; excited states 


\section{Introduction}

Molecular two-photon absorption ${ }^{1-4}$ (TPA) has attracted growing attention over recent years, thanks to the wide range of applications it offers in the fields of material science and biology. These include two-photon laser scanning microscopy (TPLSM) imaging, ${ }^{5-6}$ localized photodynamic therapy (PDT), ${ }^{7-8} 3 \mathrm{D}$ optical data storage, ${ }^{9}$ microfabrication, ${ }^{10}$ optical power limitation. ${ }^{11}$ More recently this field has encompassed new nanobiophotonic applications ${ }^{12}$ including those based on selective photoaddressing in biological environment such as synchronization of enzymatic processes in proteins and NO production via two-photon induced electron transfer by a biphotonic nanotrigger, ${ }^{13}$ or bioimaging in living animals ${ }^{14-15}$ or functional imaging in neurons. ${ }^{16}$

This has prompted the design of compounds which exhibit enhanced TPA crosssections along with specific features depending on the desired application. It should be stressed, for instance, that the 3-D resolution advantage can only be achieved if two-photon excitation is realized without concomitant one-photon excitation. In practice, this excludes the use of TPA materials whose large TPA responses are related to strong one-photon resonant enhancement. On the other hand, bioimaging applications would benefit from tailor-made fluorophores combining high fluorescence quantum yield $(\Phi)$, reduced phototoxicity and suitable photostability in addition to TPA cross-sections $\left(\sigma_{2}\right)$ considerably larger than those of endogenous chromophores in the biological spectral window (700-1200 nm) i.e. much larger than 1 GM. ${ }^{17}$ For biophotonic applications, giant non one-photon resonant TPA cross-sections are indeed required if efficient and selective $3 \mathrm{D}$ photo-addressing is sought after. ${ }^{13}$

The many advantages of TP excitation have triggered the molecular engineering of various molecular structures ${ }^{1-4}$ with enhanced TPA including dipolar, ${ }^{18-21}$ quadrupolar, ${ }^{22-33}$ branched $^{34-42}$ and conjugated dendritic structures ${ }^{43-44}$ and cyclic or multicyclic conjugated structures. ${ }^{45-46}$ Also multiporphyrins, ${ }^{47-48}$ fused $^{49}$ and expanded related systems ${ }^{50}$ have been shown to lead to giant TPA responses in relation with high resonant one-photon enhancement. Recently, fully organic nanodots based on the controlled confinement of a large and discrete number of optimized quadrupolar chromophores on a non-toxic dendritic platform ${ }^{51}$ have been shown to exhibit giant one-photon brilliance $(\varepsilon \Phi)$ in the visible and giant two-photon brilliance $\left(\sigma_{2} \Phi\right)$ in the NIR, outperforming those of quantum dots (QDs) ${ }^{52}$ while providing a valuable soft and eco-friendly alternative to toxic QDs. Incorporation of molecular twophoton chromophores in inorganic nanoparticles prepared by sol-gel processes ${ }^{53}$ has also proved effective for the design of hybrid organic nanoparticles with giant TPA cross-sections $\left(10^{7} \mathrm{GM}\right)^{54}$ and of interest for bioimaging purposes.

In this general framework, it is thus of major importance for both bioimaging and materials application to define general routes for the generation of libraries of modular biphotonic chromophores with structural (size, dimensionality, polarity) flexibility, enhanced TPA responses but also "customized" fluorescence features. A number of earlier studies conducted on symmetrical chromophores (quadrupoles, ${ }^{55}$ octupoles ${ }^{40,42,56-57}$ or conjugated multipolar branched structures ${ }^{58-60}$ ) have demonstrated that in such structures, localization of excitation on chromophoric subunits (followed by "hopping" between "equivalent" 
subunits $^{58}$ ) occurs prior to emission. Both quadrupoles and octupoles can thus show pronounced solvatochromic behavior as a consequence of the excitation localization on dipolar chromophoric subunits. Indeed emission stemming from strongly polarized excited states has been observed in octupolar or quadrupolar derivatives. ${ }^{39-40,55-56}$

Much attention has been paid in recent years to the optimization of symmetrically substituted chromophores leading to large TPA cross-sections. In contrast, the effect of endgroups dissymmetry on the nonlinear optical (NLO) response has only rarely been considered, ${ }^{61-62}$ although electronic dissymmetry is expected to influence transition dipole moments between ground and excited states and between different excited states. Recently, two-photon anisotropy has been investigated in symmetrical and dissymmetrical squaraines, ${ }^{61}$ fluorenes $^{62}$ and cationic polymethine dyes and interpreted in terms of few-states models. However, to our knowledge the benefit of using dissymmetry to tune (peak position) and modulate (peak amplitude and broadening) TPA properties remains largely untackled.

Based on these observations, we have been interested in investigating how the introduction of slight electronic dissymmetry in multipolar structures would affect localization of the emission. Our aim was to define engineering routes allowing both fine spectral tuning and control of emission localization while maintaining TPA efficiency. Our strategy was to introduce dissymmetry by minor modification of either the electron-donating or electron-withdrawing strength in specific chromophoric subunits. In that perspective, we have investigated two series of structurally related organic chromophores: (i) quadrupolar bisdonor $\mathrm{D}-\pi-\mathrm{D}, \mathrm{D}^{\prime}-\pi-\mathrm{D}^{\prime}$ and dissymmetrized $\mathrm{D}-\pi-\mathrm{D}^{\prime}$ derivatives built from a fluorene core; and (ii) three-branched octupolar-type derivatives built from a donating triphenylamine core $\mathrm{D}(-\pi-$ $A)_{3}, D\left(-\pi-A^{\prime}\right)_{3}$ and dissymmetrized derivative $D(-\pi-A)_{2}\left(-\pi-A^{\prime}\right)$. Spectroscopic investigations in solution include UV-Vis absorption, fluorescence, as well as two-photon excited fluorescence (TPEF) in the femtosecond regime. Special attention is paid to the influence of electronic dissymmetry on both the linear and nonlinear optical properties. Electrochemical studies were also conducted to provide additional information on the electronic interactions between electroactive end-groups. Time-dependent density-functional theory (TD-DFT) and essential-states approaches are used to complement and to rationalize experimental findings. Applications of these techniques to describe electronic structure and nonlinear spectroscopy of two-photon chromophores have been reviewed in detail in Ref. ${ }^{2}$

\section{Results and discussion}

\subsection{Synthesis}

Symmetrical $\left(\mathbf{Q 1},{ }^{33,63} \mathbf{Q 3}\right)$ and dissymmetrical $(\mathbf{Q 2})$ bis-donor quadrupolar-like chromophores were prepared simultaneously in a one-step one-pot combinatorial manner by performing Sonogashira coupling of the fluorene-cored building block $2^{33}$ in the presence of one equivalent of both iodo derivatives $\mathbf{1}^{64}$ and 3 (Scheme 1). The three different chromophores produced jointly in the reaction medium can be separated by column chromatography on silica gel. They are isolated as pure compounds in good yields, in almost 
perfect statistical distribution indicating that the iodo derivatives do not show major differences in reactivity in the Sonogashira coupling.

Symmetrical three-branched triphenylamine-cored octupolar chromophores $\mathbf{O 1}^{39}$ and $\mathbf{O 3}$ were synthesized by means of triple Heck couplings of trivinyl core $\mathbf{4 b}$ (resulting from the Wittig condensation between trialdehyde $\mathbf{4 a} \mathbf{a}^{65}$ and methyltriphenylphosphonium iodide in excess) with bromo derivatives 5 and 7, respectively (Scheme 2). Chromophores $\mathbf{O 1}$ and $\mathbf{O 3}$ were obtained with all- $E$ stereochemistry, as testified by ${ }^{1} \mathrm{H}$ NMR. The dissymmetrical octupolar compound $\mathbf{O 2}$ was prepared, with all- $E$ stereochemistry as well, in a four-step sequence from trialdehyde $\mathbf{4 a}$, which was first reacted with two equivalents of methyltriphenylphosphonium iodide, to afford dissymmetrical core 4c. Double Heck coupling of $\mathbf{4 c}$ with bromo derivative 5 gave aldehyde $\mathbf{6 a}$, which was converted into alkene $\mathbf{6 b}$. The latter was finally reacted with bromo derivative 7 to afford $\mathbf{O 2}$ (Scheme 2). All compounds were fully characterized by ${ }^{1} \mathrm{H} \mathrm{NMR},{ }^{13} \mathrm{C} \mathrm{NMR}$, elemental analyses and/or HRMS.

\subsection{Molecular geometry}

Quantum Mechanical (QM) calculations, performed as described in the experimental section, indicate that quadrupolar-type chromophores Q1-Q3 have quasi-identical groundstate optimized geometries, characterized by a fully planar backbone (Fig. 1). All compounds retain nearly symmetrical banana-shaped ${ }^{33}$ conjugated backbones that lead to small groundstate dipole moments $(\sim 1.5 \mathrm{D})$ pointing perpendicularly to the molecular long axis within the molecular plane. Small differences in bond lengths are observed in Q2, evidencing a slight dissymmetry between the two arms. The largest difference is observed for the C-N bond length, respectively 1.382 and $1.376 \AA$ for $\mathrm{NMe}_{2}$ and $\mathrm{NH}_{2}$ substituents. These values are identical to those obtained for their symmetrical counterparts (i.e. Q1 and Q3 quadrupoles).

The octupolar-type compounds O1-O3 adopt a propeller-shaped geometry. The phenyl rings of the triphenylamine core are twisted by $45^{\circ}$ in the symmetrical compounds $\mathbf{O 1}$ and $\mathbf{O 3}$ whereas in compound $\mathbf{O 2}$, they show a slight dissymmetry with a twist increased by $4^{\circ}$ for the branch bearing the $\mathrm{SO}_{2} \mathrm{CH}_{2} \mathrm{CH}_{2} \mathrm{OH}$ end-group with respect to those bearing the $\mathrm{SO}_{2} \mathrm{CF}_{3}$ endgroups. The phenylene-ethynylene spacers introduce an additional twist (Fig. 2).

Excited-state optimized geometries of O1-03 evidence a clear symmetry breaking; The geometry of two of the three branches remains close to their ground-state geometry with a reduced twist at the phenylene-ethynylene spacers and only a slight reduction of corresponding bond-length alternation (BLA) (from 0.14 to $0.13 \AA$ ). At opposite, the third branch becomes planar and has vanishing BLA in the excited state as observed in many extended molecular systems such as conjugated polymers. We stress that the lowest-energy relaxed excited state of chromophore $\mathbf{O 2}$ corresponds to localization occurring on one of the branches bearing the strongest electron-withdrawing peripheral groups.

Whereas a clear-cut symmetry breaking is obtained by calculations of relaxed excitedstate geometries of the octupolar compounds, no such behavior is predicted from calculations conducted on quadrupolar compounds. While dissymmetry of $\mathbf{Q 2}$ in its excited state is comparable to that obtained for its ground-state geometry, $\mathbf{Q 1}$ and $\mathbf{Q 3}$ remain symmetrical. In addition, BLA for the $\mathrm{C}-\mathrm{C} \equiv \mathrm{C}-\mathrm{C}$ unit is only slightly reduced (from 0.23 to $0.19 \AA$ ) in the excited state. This predicted difference between quadrupolar and octupolar derivatives might 
be explained taking advantage of the general results suggested by the few-state models on quadrupolar and octupolar chromophores. ${ }^{55,57}$ Indeed, those essential models predict that symmetry breaking is unconditional for octupolar chromophores, i.e. all compounds having $\mathrm{D}(-\pi-\mathrm{A})_{3}$ or $\mathrm{A}(-\pi-\mathrm{D})_{3}$ motif should have their first excited state characterized by three equivalent minima. ${ }^{57}$ On the contrary, symmetry breaking is conditional for quadrupolar chromophores: only those having small quadrupolar character and quite high electronvibration coupling will feature two minima in their first excited state. ${ }^{55}$

In view of these considerations, it is possible to suppose that symmetry breaking in the relaxed excited state is not predicted by QM calculations for the quadrupolar chromophores because the condition required for the observation of this phenomenon is not fully met. This could be related, e.g., to the fact that solvation effects, thermal bath, and inhomogeniety are not accounted for.

\subsection{Electrochemical properties}

Electrochemical properties of chromophores Q1-Q3 and $\mathbf{0 1 - 0 3}$ were determined using cyclic voltammetry (CV).

\section{Quadrupolar type chromophores:}

All compounds exhibit a reversible oxidation wave (Fig. 3), whose normalized peak currents $i_{\mathrm{p}} v^{-1 / 2}$ are independent on the scan rate $v$. The shape and the reproducibility of these signals are strongly influenced by the nature of the electrode material ( $\mathrm{Pt}, \mathrm{Au}$, glassy carbon). The absolute values of the number of electrons transferred at this step were determined using one-electron current of ferrocene and combining voltammetry and chronoamperometry data. ${ }^{66}$ Both methods suggest that the first electron transfer is monoelectronic, leading to a radical cation species (Table 1). The CV scan is reversible in agreement with the reduction of the radical cation by electron uptake during the back reduction step. UV-Vis spectroelectrochemistry of $\mathbf{Q 1}$ indeed provides evidence of the formation of an electrogenerated cation radical showing a characteristic absorption band in the visible region

(maximum at $\lambda_{\max }$ abs $\sim 575 \mathrm{~nm}$ ). Q1 oxidation shows reversibility already at low scan rates ( $v$ $\geq 0.01-0.02 \mathrm{~V} \mathrm{~s}^{-1}$ ), and an EPR signal of the radical cation is observed upon oxidation at the potentials starting from $0.45 \mathrm{~V}$ in a $0.1 \mathrm{M} \mathrm{Bu}_{4} \mathrm{NPF}_{6}-\mathrm{THF} / \mathrm{CH}_{3} \mathrm{CN}$ (4:1) solution. Three rays with $g$-factor of $2.0036 \mathrm{G}$, broadened by exchange interactions, show one nitrogen constant of $11 \mathrm{G}$. Separation of any hyperfine coupling constants from aromatic protons was not possible even at the microwave power of $0.2 \mathrm{~mW}$ and modulation amplitudes as small as $0.05 \mathrm{G}$. These observations suggest a substantial spin delocalization on the $\mathrm{N}$ atoms in the radical cation and are in line with high orbital coefficients on nitrogens.

The oxidation potential of the quadrupolar derivative bearing weaker end-groups (i.e. Q3, Table 1: $\mathrm{E}^{0}=0.496 \mathrm{~V}$ vs. $\left.\mathrm{Ag} / \mathrm{Ag}^{+}\right)$is similar to that determined for $N, N$-diethylaniline (i.e. isolated stronger end-groups) in similar conditions $\left(0.48_{6} \mathrm{~V} v \mathrm{vs} . \mathrm{Ag} / \mathrm{Ag}^{+}\right)$. In addition, $\mathrm{E}_{\mathrm{p}}{ }^{\mathrm{ox}}$ increases from $\mathbf{Q 3}$ to $\mathbf{Q 1}$, i.e. with increasing end-groups donating strength. This can be related to the quadrupolar nature of the chromophores. A slight intramolecular charge transfer from the peripheral groups to the fluorenyl core occurs in the ground state, ${ }^{67}$ resulting in a slight decrease of the electron density on the peripheral moieties which in turn increases the 
oxidation potential. This effect is more pronounced for the strongest donating end-groups ${ }^{67}$ (i.e. Q1). To some extent, variation of the oxidation potential in such series of bis-donor derivatives ${ }^{68}$ can thus be considered as a probe of the electronic quadrupolar charge transfer in the ground state.

\section{Octupoles:}

The oxidation of octupolar-type derivatives O1-O3 occurs at slightly less positive potentials than Q1-Q3 (Table 1) and at much lower potential than that of their triphenylamine core moiety (whose oxidation potential measured in similar conditions is $0.72 \mathrm{~V} \mathrm{vs.} \mathrm{Ag} / \mathrm{Ag}^{+}$). This somewhat counter-intuitive trend can be related to the radical cation stabilization by delocalization of the radical on the conjugated arms bearing peripheral groups (Scheme 3). The stronger acceptor end-groups lead to lower oxidation potential consistent with a stabilization of the radical by electron-withdrawing end-groups. ${ }^{69}$ All compounds exhibit a reversible one-electron oxidation $\mathrm{with} \mathrm{i}^{\mathrm{c}} / \mathrm{i}^{\mathrm{a}}$ ratio close to unity even at slowest scan rates (Fig. 4). A marked adsorptional activity of these compounds accounts for the somewhat larger peak widths rather than slow electron transfer. In contrast, the reduction processes are found to be irreversible leading to one-electron $\left(\mathrm{n}^{\text {red }} \cong 1\right.$ ) irreversible reduction peaks (Table 1).

\subsection{Photophysical properties}

\subsubsection{One-photon absorption (OPA)}

Experiments and QM calculations indicate that all chromophores show two main absorption bands in the near UV - visible region. The lowest-energy, more intense band, lies in the near UV region for the quadrupolar type chromophores and in the visible blue region for the octupolar type chromophores (Tables 2-3). The second, less intense absorption band observed in the UV region for all chromophores has significant intensity only for octupolar chromophores (Figs. S1,S2). In the symmetrical chromophores (Q1, Q3, O1 and O3), TDB3LYP results indicate that these two peaks correspond to transitions to the first and fifth excited state, respectively. For the octupolar compounds it should be noticed that both of these states are doubly degenerate for symmetry reasons. Intermediate states have very small or vanishing oscillator strengths, especially state $\mid 2>$ for quadrupoles (Fig. 1) and $\mid 3>$ for octupoles (Fig. 2). These states become partially OPA allowed for dissymmetrical chromophores Q2 and O2. Since symmetry in chromophores Q2 and $\mathbf{O 2}$ is only slightly lowered, the corresponding induced OPA oscillator strengths remain weak. This results in a slight broadening at the blue edge of the lower-energy absorption band (Figs. S1, S2). Dissymmetry is also clearly visible on the natural transition orbitals of $\mathbf{O 2}$ (Fig. 2).

As expected, we observe a bathochromic and hyperchromic shift of the first absorption band with increasing strength of the peripheral end-groups for both the quadrupolar-type and octupolar-type chromophores (Tables 2-3) consistent with an intramolecular charge transfer from the periphery to the core in the case of the quadrupoles, and from the core to the branches in the case of the octupoles, as clearly evidenced from the natural transition orbitals (Figs. 1-2). As a result, the absorption band maxima for the dissymmetrical compounds (Q2, O2) are located in between those of their symmetrical analogues (Tables 2-3), allowing fine 
spectral tuning of the absorption properties. Notice that even though state $\mid 1>$ of $\mathbf{O} 2$ is slightly red shifted with respect to that of $\mathbf{O 1}$, close proximity of state $\mid 2>$ leads to an overall blue shift of the first absorption band.

\subsubsection{Fluorescence}

\subsubsection{Quadrupoles}

Quadrupolar-type chromophores Q1-Q3 show an intense emission band in the violet visible region in toluene with high quantum yields and fluorescence lifetimes smaller than one nanosecond (Tables 2-3). Fluorescence quantum yields and lifetimes compare well with those reported for related rod-like and banana-shaped quadrupolar fluorophores. ${ }^{33}$ As expected, a red shift of the emission band is observed with increasing donating strength of the end-groups for the symmetrical compounds. The radiative decay rate is not affected, indicating that the red shift of the emission is compensated by an increase in the transition dipole between the emitting excited state and ground state, which parallels the hyperchromic shift of the absorption band (Tables 2-3). The non-radiative decay rate decreases with increasing donating strength, most probably in relation with the nature of the end-groups $\left(\mathrm{NH}_{2}\right)$ rather than their strength. As a result, symmetrical chromophore Q1 with the strongest end-groups has the highest fluorescence quantum yield.

The behavior of dissymmetrical derivative $\mathbf{Q 2}$ is interesting (Tables 2-3). Whereas the low-energy absorption band is located in between that of the two symmetrical parent quadrupoles. However, the emission band of quadrupole Q2 is close to that of quadrupole Q1 bearing the strongest end-groups, indicating that the emitting excited states have in Q1 and Q2 some similarities. On the other hand, dissymmetrical chromophore Q2 shows lower radiative decay rate than both symmetrical chromophores $\mathbf{Q 1}$ and $\mathbf{Q 3}$. This can be related to the red shift of the emission of $\mathbf{Q 2}$ as compared to $\mathbf{Q 3}$ and indicates that the transition dipole between emitting excited state and ground state of chromophore $\mathbf{Q 2}$ is slightly lower than that of chromophore Q1 (Table 3) since both of them have close emission wavelengths. In contrast the non-radiative decay rate of chromophore Q2 is similar to that of chromophore Q3, suggesting that non-radiative vibrational channels involving $\mathrm{NH}_{2}$ end-groups are involved in both cases. Experimental and calculated photophysical properties summarized in Tables 2 and 3 , respectively, show a good overall agreement.

\subsubsection{Octupoles}

Octupolar-type chromophores O1-03 show an intense emission band in the blue visible region in toluene with high fluorescence quantum yields. The emission characteristics (lifetime and quantum yields) are similar to those of related octupolar derivatives. ${ }^{39}$ Octupolar-type chromophores O1-O3 show significantly longer fluorescence lifetimes (about $2 \mathrm{~ns}$ ) than quadrupolar-type derivatives Q1-Q3 while having similar fluorescence quantum yields. This striking behavior can be related to smaller radiative and non-radiative decay rates (Table 2). We observe that the radiative decay rates of octupolar-type derivatives O1-O3 are more than twice smaller than those of quadrupolar type derivatives Q1-Q3 which can be in partially attributed to the red-shifted emission of investigated octupoles as compared to 
quadrupoles (Table 2). Furthermore, calculated transition dipole moments between an emitting state and the ground state is larger in octupolar molecules compared to quadrupoles (Table 3), which is also attributed to the difference in the radiative decay rates.

As was observed in the case of quadrupolar-type derivatives, the stronger end-groups lead to a red shift of the emission band of octupolar derivatives (O1 versus O3). Interestingly the two derivatives show similar radiative and non-radiative decay rates, and thus similar fluorescence lifetimes and quantum yields. The dissymmetrical octupolar-type chromophore $\mathbf{O 2}$ shows interesting features as was the case for quadrupolar-type chromophore Q2. Its emission band is similar to that of the octupolar chromophore bearing the strongest electronwithdrawing peripheral groups (O1, Tables 2-3) indicating that the emitting excited states for the two different chromophores are similar. We note that radiative decay rate of chromophore $\mathbf{O 2}$ is slightly higher than that of related symmetrical chromophores $\mathbf{O 1}$ and $\mathbf{O 3}$ although its emission is red-shifted (Table 2), suggesting that the transition dipole between the emitting excited state and the ground state is slightly larger in the dissymmetrical derivative.

\subsubsection{Solvent effects. Nature of the emitting excited states}

The solvatochromic behavior of chromophores Q1-Q3 and chromophores O1-O3 is reported in Figures 5 and 6. All compounds show a weak positive solvatochromic absorption behavior and a much more pronounced positive solvatochromic emission behavior, corresponding to a marked red-shift of the emission band with increasing polarity. Such behavior is indicative of polar emitting states and has already been reported previously for related quadrupolar ${ }^{27,70}$ and octupolar type chromophores. ${ }^{39,71}$ The absorption behavior of purely quadrupolar and octupolar chromophores is easily understood based on symmetry reasons: the states involved in the transition have no significant permanent ground-state dipole moment, so that the transition energy is expected to be only weakly dependent on the solvent polarity. This is indeed observed for spectra of Q1, Q3, O1 and O3, with very weak dependence of the transition energy on the solvent polarity. This dependence could be ascribed to small dipole moments due to slight deviation from perfect symmetry (in particular for quadrupoles which are not truly centrosymmetrical, and due to conformational disorder), to higher multipole effects or to the different refractive indexes of the solvents. The same behavior is also observed for dissymmetrical compounds (Q2 and O2) suggesting that the different end-groups do not generate major symmetry breaking in the ground state.

The fluorescence process has completely different characteristics. Emission stems from the relaxed excited state, where vibrational and solvation degrees of freedom are equilibrated with the electronic charge distribution of the excited state. The strong solvatochromism of the fluorescence emission is consistent with a symmetry breaking in the relaxed excited state, corresponding to the localization of the excitation on a portion of the chromophores leading to polar emitting excited states.

Indeed, calculations of optimized excited state geometries and related NTOs indicate that octupolar-type derivatives O1-O3 show excitation localization on a single branch (Fig. 7). Excitation localization in branched systems is not uncommon and has been reported in various branched systems built from a triphenylamine core ${ }^{40,58}$ or different cores. ${ }^{42,56,59-60}$ In the case of octupolar derivatives, excitation localization on single branches occurs after 
excitation prior to emission for any (even slightly polar) solvent, because of the unconditional nature of symmetry breaking for these chromophores. ${ }^{55}$ This is the reason why we observe that the emission solvatochromism of the octupolar-type derivatives O1-03 follows the Lippert-Mataga relationship with a linear dependency of the Stokes shift as a function of a polarity/polarizability parameter ${ }^{72-73}$ (Fig. 8):

$$
\widetilde{v}_{a b s}-\widetilde{v}_{e m}=2 \Delta \mu^{2} \Delta f /\left(h c a^{3}\right)+\text { const }
$$

where $\widetilde{v}_{a b s}$ and $\widetilde{v}_{e m}$ are the wavenumbers of the absorption and fluorescence maxima, $h$ is the Planck constant, $c$ is the light velocity, $\Delta \mu$ is the change of dipole moment between the ground state and the emitting excited state and $\Delta f$ is defined as:

$$
\Delta f=(\varepsilon-1) /(2 \varepsilon+1)-\left(n^{2}-1\right) /\left(2 n^{2}+1\right)
$$

where $\varepsilon$ is the dielectric constant and $n$ is the refractive index of the solvent.

We observe that chromophore $\mathbf{O 2}$ shows almost identical solvatochromic behavior as chromophore 01 (Fig. 6). Furthermore the Lippert-Mataga slopes, which have close values for the different chromophores, are similar for $\mathbf{O 1}$ and $\mathbf{O 2}$ (Fig. 8), suggesting that emission preferentially originates from one of the branches bearing the strongest electron-withdrawing end-groups. On the other hand, we observe that $\mathbf{O 2}$ shows the highest Stokes shift values whatever the solvent, indicating larger nuclear/solvent reorganization after excitation prior to emission.

The quadrupolar-type derivatives Q1-Q3 show a slightly different behavior. The emission solvatochromism is found to obey the Lippert Mataga relationship in medium to high polarity environments (Fig. 8) whereas the experimental data obtained for very low polarity solvent (toluene) clearly deviates from the linear dependence. In addition, calculations of optimized excited state geometries and related NTOs (Fig. 9) do not reveal excitation localization but rather emission originating from the whole conjugated structure for the isolated molecule. This suggests that whereas no localization is observed in the isolated molecules or in an apolar environment (false symmetry breaking), ${ }^{57}$ a true symmetry breaking is observed in weak to high polarity environment. ${ }^{55}$ Interestingly as compared to recent data reported for different quadrupolar derivatives built from a xylene or anthracene core ${ }^{70}$ we do not observe a bilinear Lippert-Mataga plot, with low slope values in low polarity environments and high slope values for high polarity environments. This indicates that the symmetry breaking onset occurs for lower solvent polarity in the case of the present quadrupolar systems built from a fluorene core, indicative of stronger electron-phonon coupling. Interestingly we observe that chromophore Q2 shows similar emission behavior (Fig. 5) as chromophore Q1, indicative that emission localizes on a common portion of the molecule with respect to $\mathbf{Q 1}$. This suggests that $\mathbf{Q} 2$ emission stems mainly from a part of the molecule bearing the strongest electron-donating end-group.

From the Lippert-Mataga plots, one can derive the $\Delta \mu$ values and thus get an estimation of the dipole moment of the emitting excited state for all chromophores provided an accurate estimation of the cavity radius is available. Following Rebane and co-workers, ${ }^{74}$ we have investigated the variation of the fluorescence emission anisotropy (and lifetime) as a function of solvent characteristics to derive the $a$ values using the Perrin equation: ${ }^{75}$

$$
r=0.4 /(1+\mathrm{k} T \tau / \eta \mathrm{v})
$$


where $r$ is the anisotropy ratio, $\tau$ the fluorescence lifetime, $\mathrm{v}$ the molecular volume, $\eta$ the solvent viscosity and $T$ the temperature.

As shown in Figure 10, a linear dependence of $0.4 / \mathrm{r}-1$ as a function of $\tau / \eta$ is observed for the quadrupolar-type chromophores, the slope values yielding estimated radius values of respectively 10.6, 9.1 and $8.3 \AA$ for $\mathbf{Q 1}, \mathbf{Q 2}$, and $\mathbf{Q 3}$ respectively. From the slope values of the Lippert-Mataga plots one can then derive excited state dipole moment values of respectively, 18, 15 and 12D. Hence as was reported earlier in octupolar systems derived from triphenylamine core, ${ }^{39}$ stronger end-groups lead to more polar emitting excited states in symmetrical quadrupolar chromophores too. This behavior - which is similar to that reported for related dipolar chromophores ${ }^{76-77}$ or push-pull polyenes ${ }^{78-79}$ - is a further confirmation of excitation localization on dipolar subunits in the excited states of multipolar structures. Interestingly, the dissymmetrical derivative $\mathbf{Q} \mathbf{2}$ has intermediate excited state dipole moment as compared to parent symmetrical quadrupoles. Hence dissymmetry allows tuning the polarity of the emitting excited state.

In contrast to rod-like quadrupolar derivatives, steady-state anisotropy experiments performed on three-branched octupoles yield low anisotropy ratio values (typically 0.02 in toluene for $\mathbf{O 1}$ while $\mathbf{Q 1}$ value is about ten times larger in the same solvent). This behavior can be related to their different geometry and the fact that excitation localization after absorption prior to emission (i.e. in the relaxed emitting excited state) may occur on different branches, thus resulting in a reduction of emission anisotropy. Such process has been reported to be very fast in branched systems including octupoles built from a triphenylamine core ${ }^{58}$ leading to major anisotropy loss.

\subsubsection{Simulations of solvent effect in the essential state approach}

The solvatochromic behavior for quadrupolar and octupolar chromophores has been recently rationalized in the framework of few-states models. ${ }^{55,57}$ These models account for 3 and 4 electronic basis states for quadrupoles and octupoles, respectively, linear coupling to 2 and 3 effective molecular vibrations, respectively, and dipolar solvation (see Experimental section). A conditional bistability is predicted in the first (i.e. one-photon active) excited state for quadrupolar chromophores, according to the strength of electron-phonon coupling, and symmetry breaking is induced by relaxation of the solvation coordinate. For octupolar dyes instead, multistability in the first excited state is unconditional, so that a dipolar solvent always breaks the symmetry of the emitting excited state. The molecular parameters of these few-state models can be optimized in order to reproduce spectral properties, such as absorption and fluorescence (with corresponding solvatochromism) and TPA (if available). Results obtained for absorption and fluorescence spectra of the quadrupolar and octupolar chromophores studied in this paper are shown in Figures 5 and 6 , respectively. The main characteristics of the spectra are well reproduced, including fluorescence solvatochromism, inhomogeneous broadening effects, as well as the evolution of the vibronic structure (for quadrupoles). In particular, quadrupolar chromophores Q1 and Q3 belong to class I, as defined in Ref. ${ }^{55}$, i.e. the potential energy surface relevant to the first excited state is bistable, having two equivalent minima corresponding to deformation of the molecule along one branch or the other. For octupolar chromophores, three minima are obtained, as it is always 
the case because of degenerate states. In the case of multistable potential energy surfaces, the relaxed (emitting) excited state can be either delocalized between the multiple minima (false symmetry breaking), or localized on one of them (true symmetry breaking). But in any case, in the presence of a polar solvent, symmetry will be effectively broken, corresponding to the stabilization of one single minimum, i.e. localization of the excitation on the relevant branch. This is the origin of the solvatochromic behavior observed in the fluorescence spectra of all octupolar compounds, and of quadrupolar chromophores belonging to class I.

Description of the solvatochromism of dissymmetrical compounds (Q2 and O2) can be done by using the same models, but assigning a different energy value to one of the zwitterionic basis states with respect to the other(s). In the case of our chromophores $\mathbf{Q 2}$ and O2, differences are small, so that deviations from perfect symmetry are weak. The behavior of Q2 and $\mathbf{O 2}$ is then very similar to those of the symmetrical analogues, with delocalized vertical excited states (weakly dissymmetrical) and localized relaxed (emitting) excited states (at least in medium to high polarity solvents for quadrupoles). As already stated above, emission stems from the branches bearing the strongest donor/acceptor group(s) for Q2/O2, as suggested by the close resemblance of emission transition energies of Q2 and Q1, as well as of $\mathrm{O2}$ and $\mathbf{0 1}$. Particularly interesting is the behavior of the vibronic progressions in fluorescence spectra, evident in the case of quadrupolar derivatives. While the Franck-Condon structure of the two purely quadrupolar $\mathbf{Q 1}$ and $\mathbf{Q 3}$ compounds is very similar, the vibronic progression of $\mathbf{Q} 2$ is different, with the 0-1 vibronic band gaining intensity with respect to the 0-0 line. This behavior is well reproduced by calculated spectra, with no need of any ad hoc change in the electron-phonon coupling constants. This observation is hindered in the case of octupolar compounds because the vibronic structure is smeared out, the origin of which probably lying in the presence of very low-frequency torsional degrees of freedom. These might be directly related to the different nature of the core (planar rigid fluorene core for quadrupoles Q1-Q3 and propeller-shaped triphenylamine core for octupoles O1-O3) or of the spacers (phenylene-ethynylene versus phenylene-vinylene).

\subsection{Two-photon absorption}

TPA spectra of all chromophores have been determined through femtosecond twophoton excited fluorescence experiments in toluene and ethanol for quadrupoles and octupoles, respectively, for solubility reasons. Excited state quantum-chemical calculations and essential-state approaches (described in the experimental and computational details section) were also performed to interpret experimental data. Neither solvent effects nor vibrational contributions have been taken into account in the quantum-chemical calculations. On the contrary, both these effects are introduced in the essential-state analysis, referring to toluene as solvent, even for octupoles, because of computation time. Despite these differences, the overall agreement between these theoretical approaches is good (Fig. 11 and 12). Differences observed in the shorter wavelengths range can be related to higher lying excited states not accounted for in the essential-state description. The calculated absolute TPA magnitudes and the respective experimental values agree roughly within a factor of two (note 
that position and amplitude of the main TPA maxima in Q2 and Q3 chromophores are not available from experiment) This seems reasonable given the number of approximations in theory ${ }^{2,55,57}$ and the margins of error in experiment. ${ }^{2}$

Quadrupoles: The first TPA maximum is not reached in the spectral window investigated by experiment (Fig. 11). Indeed QM calculations predict that this maximum should appear at wavelengths shorter than $700 \mathrm{~nm}$. The spectral blue-shift when going from Q1 to Q3 is consistent with that observed in the OPA spectra, and is confirmed by the calculations, where the first TPA maxima are easily accessible. As expected for quadrupolar systems, ${ }^{67,80}$ this maximum shows up at higher energy and thus at wavelength smaller than twice the onephoton maximum. Even if quadrupolar compounds Q1-Q3 do not have perfect inversion symmetry, their lowest excited state is predominantly one-photon allowed while the second excited state is predominantly two-photon allowed. The latter corresponds to definite quadrupolar charge redistribution from the periphery to the fluorene core upon photoexcitation (Fig. 1), which is responsible for the high TPA responses. These responses compare well with those observed in related rod-like and banana-shaped quadrupolar fluorophores. ${ }^{27,33,81}$ Substitution of only one of the strong donating end-groups $\left(\mathrm{NHex}_{2}\right)$ by a weaker donating end-group $\left(\mathrm{NH}_{2}\right)$ has only small effect on the magnitude of the TPA maximum. In contrast, larger variations of the TPA responses are observed in the low energy region (which is the experimentally accessible region) as a result of the red-shift of the TPA bands with increasing electron-donating strength. Quadrupolar chromophores Q2 and Q3 hence show much lower TPA responses in the 700-750 nm region than chromophore Q1 because this corresponds only to the onset of the intense TP allowed absorption band for the former chromophores. We however observe that in the region corresponding to the transition to the lowest one-photon allowed excited state (750-800 nm region) supposedly two-photon forbidden, chromophore $\mathbf{Q} 2$ shows comparable TPA magnitude as compared to chromophore Q1 whereas chromophore Q3 has a very low response. This can be related to the slight electronic dissymmetry imparted in chromophore $\mathbf{Q 2}$.

Octupoles: TPA spectra of the octupolar compounds O1-O3 present features similar to those discussed for the quadrupolar systems with the advantage of having their first TPA maximum within the experimentally accessible spectral window (Fig. 12). Substitution of the electronwithdrawing end-group $\mathrm{SO}_{2} \mathrm{CF}_{3}$ by a weaker acceptor $\mathrm{SO}_{2} \mathrm{CH}_{2} \mathrm{CH}_{2} \mathrm{OH}$ only slightly reduces the TPA maximum amplitude. The spectral blue-shift is again consistent with that observed for the OPA spectra, and results in larger variations of the TPA response in the NIR spectral region. As a consequence of significant band broadening in the low energy part of the spectrum, the dissymmetrical compound $\mathbf{O 2}$ maintains comparable TPA performances to those achieved by octupole $\mathbf{0 1}$. This behavior is well reproduced by QM calculations, as well as by results obtained in the framework of the essential-state model. The band broadening in $\mathbf{O 2}$ is directly caused by the loss of $\mathrm{C}_{3}$ symmetry. Indeed, for fluorophores of $\mathrm{C}_{3}$ symmetry the first two excited states are degenerate and OP-allowed. Their low two-photon activity leads to a shoulder at the wavelength of around twice the OPA maximum (Fig. 12). Their third excited state is nearly OPA-forbidden and mainly responsible for the amplitude of the 
first TPA peak. For the symmetrical octupolar systems $\mathbf{O 1}$ and $\mathbf{0 3}$, the large TPA responses come from the marked core to periphery charge redistribution upon photoexcitation which is illustrated in Fig. 2. Dissymmetry in fluorophore $\mathbf{O 2}$ breaks the excited-state degeneracy, and the corresponding one and two-photon oscillator strengths are redistributed among the different excited states. While the OPA oscillator strengths of the first, third and fourth excited states increase, that of the second excited state decreases (Fig. 2). The increase in the two-photon absorptivity of the latter is mainly responsible for the broadening at the lowenergy edge of the calculated and experimental TPA spectra.

Introduction of dissymmetry in both quadrupolar and octupolar systems (Q2, O2) induces a spectral shift bringing the TPA maximum in between those of the two corresponding symmetrical chromophores (Q1 and Q3; O1 and O3). Symmetry breaking induced by the presence of the two different end-groups also leads to spectral broadening. While this is clearly visible for the octupolar fluorophores, the effect is not so pronounced for quadrupolar compounds as a consequence of the small difference in donor strengths and the absence of true inversion symmetry in the related symmetrical quadrupoles (which also explains why chromophore Q1 shows a weak TPA band in the 750-800 nm region). In fact, two-photon forbidden (or slightly allowed) excited states become two-photon allowed due to dissymmetry because of oscillator-strength redistribution among excited states. It should be noted that TPA is a nonlinear process and thus the effect of oscillator strength redistribution cannot be thought of as a linear process and may lead to nontrivial behavior.

\section{Conclusion}

Symmetrical banana-shaped quadrupolar and propeller-shaped octupolar derivatives bearing different end-groups and their dissymmetrical analogues have been synthesized and investigated in order to assess the effects of slight dissymmetry on both TPA responses and emission properties. Dissymmetry has been obtained by replacing one of the end-groups by a slightly weaker donor in the case of push-push quadrupoles or alternatively one of the peripheral groups by a slightly weaker acceptor for the octupoles built from a triphenylamine core. Investigation of their linear and nonlinear optical properties reveals that slight dissymmetrical functionalization offers interesting perspectives allowing both spatial and spectral control of the fluorescence properties: while a delocalized intramolecular charge transfer takes place upon excitation, fluorescence stems from an excited state located on one of the molecular subunits bearing the strongest end-groups. In addition the electronic dissymmetry induces spectral shifts that can be exploited to tune one- and two-photon absorption spectra. These findings are corroborated by theoretical and QM calculations, shedding light on the symmetry-breaking behavior of these compounds. The present study shows that the more intense TPA maxima only slightly decrease while spectral broadening is observed as a result of dissymmetry. This in turn leads to enhanced TPA responses in specific spectral regions where formerly two-photon forbidden (or only weakly allowed) excited states become two-photon allowed. This results directly from a redistribution of the oscillator strengths among the excited states, which is caused by the degeneracy loss consequent to symmetry breaking. Because slight electronic dissymmetry is responsible for these effects, 
such modification of TPA responses is expected to occur also when symmetrical compounds are positioned in dissymmetrical environments (e.g., at interfaces). Such effect could be of particular interest for probing interfaces via modification of the TPA responses of biphotonic probes in the spectral region where the TPA responses are more affected. Monitoring chromophore adsorption and orientation at interfaces should be possible by following the change in the TPA responses in this spectral region. Another particularly interesting promise relies on the spatial control of the emission localization at the submolecular level in formerly symmetrical structures. This could tentatively be extended for the directional control at the nanoscale level of processes such as energy or electron transfer and opens an interesting route for the design of smart nanoarchitectures for energy harvesting.

\section{Experimental and computational details}

\section{Synthesis of the chromophores}

Synthesis of quadrupoles Q1-Q3. Air was removed from a solution of $N, N$-dihexyl-4iodobenzenamine (1) $)^{64}(205 \mathrm{mg}, 0.530 \mathrm{mmol})$, 4-iodoaniline (3) $(116 \mathrm{mg}, 0.530 \mathrm{mmol})$ and 2,7-diethynyl-9,9-dinonyl-9H-fluorene $(2)^{33}(247 \mathrm{mg}, 0.530 \mathrm{mmol})$ in $5 \mathrm{~mL}^{\circ} \mathrm{ot}_{3} \mathrm{~N}$ by blowing argon for $20 \mathrm{~min}$. Then $\mathrm{CuI}(4.0 \mathrm{mg}, 0.021 \mathrm{mmol})$ and $\mathrm{Pd}\left(\mathrm{PPh}_{3}\right)_{2} \mathrm{Cl}_{2}(14.9 \mathrm{mg}, 0.021$ $\mathrm{mmol}$ ) were added, and deaeration was continued for $10 \mathrm{~min}$. Thereafter the mixture was stirred at $35{ }^{\circ} \mathrm{C}$ for $15 \mathrm{~h}$. The solvent was removed under reduced pressure, and the crude product was purified by column chromatography (heptane/ $\mathrm{CH}_{2} \mathrm{Cl}_{2} 90: 10,60: 40$ then $25: 75$ ) to yield $138 \mathrm{mg}$ (26\%) of Q1, $203 \mathrm{mg}$ (47\%) of Q2 and $72 \mathrm{mg}$ (21\%) of Q3.

4,4'-[(9,9-Dinonyl-9H-fluorene-2,7-diyl)di-2,1-ethynediyl]bis( $N, N$-dihexylbenzenamine) (Q1). ${ }^{1} \mathrm{H}$ NMR $\left(200.13 \mathrm{MHz}, \mathrm{CDCl}_{3}\right) \delta 7.60(\mathrm{~d}, J=8.3 \mathrm{~Hz}, 2 \mathrm{H}), 7.46(\mathrm{~d}, J=8.3 \mathrm{~Hz}, 2 \mathrm{H})$, $7.45(\mathrm{~s}, 2 \mathrm{H}), 7.39$ and $6.57\left(\mathrm{AA}^{\prime} \mathrm{XX}^{\prime}, J_{\mathrm{AX}}=9.0 \mathrm{~Hz}, 8 \mathrm{H}\right), 3.27(\mathrm{~m}, 8 \mathrm{H}), 1.96(\mathrm{~m}, 4 \mathrm{H}), 1.58(\mathrm{~m}$, $8 \mathrm{H}), 1.32(\mathrm{~m}, 24 \mathrm{H}), 1.30-0.99(\mathrm{~m}, 24 \mathrm{H}), 0.90(\mathrm{t}, J=6.6 \mathrm{~Hz}, 12 \mathrm{H}), 0.83(\mathrm{t}, J=6.7 \mathrm{~Hz}, 6 \mathrm{H})$, $0.61(\mathrm{~m}, 4 \mathrm{H}) ;{ }^{13} \mathrm{C} \mathrm{NMR}\left(50.32 \mathrm{MHz}, \mathrm{CDCl}_{3}\right) \delta 150.9,147.8,140.0,132.8,130.3,125.5$, $122.7,119.6,111.2,108.7,91.1,88.2,55.1,50.9,40.5,31.8,31.7,30.1,29.6,29.3,29.2,27.2$, 26.8, 23.7, 22.7, 22.6, 14.1, 14.0; HRMS (LSIMS ${ }^{+}, \mathrm{mNBA}$ ) calcd for $\mathrm{C}_{71} \mathrm{H}_{104} \mathrm{~N}_{2}\left(\mathrm{M}^{+} \cdot\right) \mathrm{m} / \mathrm{z}$ 984.8200, found 984.8209. Anal. Calcd for $\mathrm{C}_{71} \mathrm{H}_{104} \mathrm{~N}_{2}$ (985.62): C, 86.52; H, 10.64; N, 2.84 . Found: C, 86.12; H, 10.85; N, 2.88.

4-[2-[7-[2-(4-Aminophenyl)ethynyl]-9,9-dinonyl-9H-fluoren-2-yl]ethynyl]-N,Ndihexylbenzenamine (Q2). ${ }^{1} \mathrm{H} \mathrm{NMR}\left(200.13 \mathrm{MHz}, \mathrm{CDCl}_{3}\right) \delta 7.63(\mathrm{~d}, J=7.5 \mathrm{~Hz}, 2 \mathrm{H}), 7.48$ $(\mathrm{d}, J=7.5 \mathrm{~Hz}, 2 \mathrm{H}), 7.47(\mathrm{~s}, 2 \mathrm{H}), 7.41$ and 6.59 (AA' $\left.\mathrm{AX}^{\prime}, J_{\mathrm{AX}}=8.9 \mathrm{~Hz}, 4 \mathrm{H}\right), 7.38$ and 6.65 $\left(\mathrm{AA}^{\prime} \mathrm{XX}\right.$ ', $\left.J_{\mathrm{AX}}=8.5 \mathrm{~Hz}, 4 \mathrm{H}\right), 3.83(\mathrm{~s}, 2 \mathrm{H}), 3.29(\mathrm{~m}, 4 \mathrm{H}), 1.97(\mathrm{~m}, 4 \mathrm{H}), 1.58(\mathrm{~m}, 4 \mathrm{H}), 1.33(\mathrm{~m}$, $12 \mathrm{H}), 1.25-1.05(\mathrm{~m}, 24 \mathrm{H}), 0.92(\mathrm{t}, J=6.4 \mathrm{~Hz}, 6 \mathrm{H}), 0.84(\mathrm{t}, J=7.1 \mathrm{~Hz}, 6 \mathrm{H}), 0.62(\mathrm{~m}, 4 \mathrm{H}) ;{ }^{13} \mathrm{C}$ NMR $\left(75.48 \mathrm{MHz}, \mathrm{CDCl}_{3}\right) \delta 150.9,147.9,146.5,140.3,139.9,132.9,132.8,130.6,130.3$, $125.6,125.5,122.8,122.2,119.7,114.7,112.7,111.2,108.7,91.2,90.3,88.5,88.2,55.1$, $50.9,40.4,31.8,31.7,30.0,29.5,29.3,29.2,27.2,26.8,23.7,22.7,22.6,14.1,14.0$; HRMS $\left(\mathrm{ES}^{+}\right)$calcd for $\mathrm{C}_{59} \mathrm{H}_{81} \mathrm{~N}_{2}\left([\mathrm{M}+\mathrm{H}]^{+}\right) \mathrm{m} / z$ 817.6400, found 817.6393.

4,4'-[(9,9-Dinonyl-9H-fluorene-2,7-diyl)di-2,1-ethynediyl]dibenzenamine (Q3). ${ }^{1} \mathrm{H}$ NMR $\left(200.13 \mathrm{MHz}, \mathrm{CDCl}_{3}\right) \delta 7.63(\mathrm{~d}, J=7.7 \mathrm{~Hz}, 2 \mathrm{H}), 7.49(\mathrm{~d}, \mathrm{~J}=7.7 \mathrm{~Hz}, 2 \mathrm{H}), 7.48(\mathrm{~s}, 2 \mathrm{H}), 7.39$ 
and $6.65\left(\mathrm{AA}^{\prime} \mathrm{XX} X^{\prime}, J_{\mathrm{AX}}=8.50 \mathrm{~Hz}, 8 \mathrm{H}\right), 3.84($ broad s, $4 \mathrm{H}), 1.97(\mathrm{~m}, 4 \mathrm{H}), 1.24-1.05(\mathrm{~m}, 24 \mathrm{H})$, $0.84(\mathrm{t}, J=7.1 \mathrm{~Hz}, 6 \mathrm{H}), 0.62(\mathrm{~m}, 4 \mathrm{H}) ;{ }^{13} \mathrm{C} \mathrm{NMR}\left(75.48 \mathrm{MHz}, \mathrm{CDCl}_{3}\right) \delta 150.9,146.6,140.2$, 132.9, 130.4, 125.6, 122.4, 119.7, 114.8, 112.7, 90.4, 88.4, 55.1, 40.4, 31.8, 30.0, 29.5, 29.3, 29.2, 23.7, 22.6, 14.0; HRMS (ES ${ }^{+}$) calcd for $\mathrm{C}_{47} \mathrm{H}_{57} \mathrm{~N}_{2}\left([\mathrm{M}+\mathrm{H}]^{+}\right) \mathrm{m} / z$ 649.4522, found 649.4523 .

Tris(4-ethenylphenyl)amine (4b). To a solution of $4 \mathbf{a}^{65}(1.1 \mathrm{~g}, 3.339 \mathrm{mmol})$ and methyltriphenylphosphonium iodide $(10.79 \mathrm{~g}, 26.7 \mathrm{mmol})$ in anhydrous THF $(25 \mathrm{~mL})$ was added sodium hydride ( $1.335 \mathrm{~g}, 60 \%$ dispersion in mineral oil). The reaction mixture was stirred for $15 \mathrm{~h}$ at $20^{\circ} \mathrm{C}$. After filtration through Celite, the crude product was purified by column chromatography $\left(\mathrm{CH}_{2} \mathrm{Cl}_{2}\right)$ to yield $1.07 \mathrm{~g}(99 \%)$ of $4 \mathbf{b} ; \mathrm{mp} 81-82{ }^{\circ} \mathrm{C} ;{ }^{1} \mathrm{H}$ NMR $(200.13$ $\left.\mathrm{MHz}, \mathrm{CDCl}_{3}\right) \delta 7.36$ and $7.11\left(\mathrm{AA}^{\prime} \mathrm{XX} X^{\prime}, J_{\mathrm{AX}}=8.6 \mathrm{~Hz}, 12 \mathrm{H}\right), 6.73(\mathrm{dd}, J=17.6 \mathrm{~Hz}, J=10.9$ $\mathrm{Hz}, 3 \mathrm{H}), 5.72(\mathrm{~d}, J=17.6 \mathrm{~Hz}, 3 \mathrm{H}), 5.23(\mathrm{~d}, J=10.9 \mathrm{~Hz}, 3 \mathrm{H}) ;{ }^{13} \mathrm{C} \mathrm{NMR}\left(75.47 \mathrm{MHz}, \mathrm{CDCl}_{3}\right)$ $\delta$ 147.0, 136.2, 132.4, 127.1, 124.1, 112.4; HRMS (EI) calcd for $\mathrm{C}_{24} \mathrm{H}_{21} \mathrm{~N}\left(\mathrm{M}^{+} \cdot\right) \mathrm{m} / \mathrm{z}$ 323.1659 , found 323.1659 .

4-[Bis(4-ethenylphenyl)amino]benzaldehyde (4c). To a solution of tris(4-formylphenyl)amine (4a) ${ }^{65}$ (500 mg, $1.518 \mathrm{mmol}$ ) and methyltriphenylphosphonium iodide (920 mg, 2.277 $\mathrm{mmol})$ in anhydrous THF $(25 \mathrm{~mL})$ was added sodium hydride $(0.175 \mathrm{~g}, 60 \%$ dispersion in mineral oil). The reaction mixture was stirred for $24 \mathrm{~h}$ at $20^{\circ} \mathrm{C}$. After filtration through Celite, the crude product was purified by column chromatography (heptane $/ \mathrm{CH}_{2} \mathrm{Cl}_{2} 50: 50$ ) to yield $154.9 \mathrm{mg}(31 \%)$ of $4 \mathrm{c} ;{ }^{1} \mathrm{H}$ NMR $\left(200.13 \mathrm{MHz}, \mathrm{CDCl}_{3}\right) \delta 9.80(\mathrm{~s}, 1 \mathrm{H}), 7.68$ and 7.10 $\left(\mathrm{AA}^{\prime} \mathrm{XX} X^{\prime}, J_{\mathrm{AX}}=8.8 \mathrm{~Hz}, 4 \mathrm{H}\right), 7.36$ and $7.05\left(\mathrm{AA}^{\prime} \mathrm{XX}{ }^{\prime}, J_{\mathrm{AX}}=8.8 \mathrm{~Hz}, 8 \mathrm{H}\right), 6.68(\mathrm{dd}, J=17.6$ $\mathrm{Hz}, J=10.9 \mathrm{~Hz}, 2 \mathrm{H}), 5.69(\mathrm{~d}, J=17.6 \mathrm{~Hz}, 2 \mathrm{H}), 5.23(\mathrm{~d}, J=10.9 \mathrm{~Hz}, 2 \mathrm{H}) ;{ }^{13} \mathrm{C} \mathrm{NMR}(50.32$ $\left.\mathrm{MHz}, \mathrm{CDCl}_{3}\right) \delta 190.4,152.9,145.6,135.9,134.4,131.3,129.6,127.6,126.0,120.1,113.8$; HRMS (EI) calcd for $\mathrm{C}_{23} \mathrm{H}_{19} \mathrm{NO}\left(\mathrm{M}^{+} \cdot\right) \mathrm{m} / \mathrm{z} 325.1467$, found 325.1461 .

\section{4-[Bis[4-[(1E)-2-[4-[(trifluoromethyl)sulfonyl]phenyl]ethenyl]phenyl]amino]-}

benzaldehyde (6a). Air was removed from a solution of $\mathbf{4 c}(76 \mathrm{mg}, 234 \mu \mathrm{mol})$ and $\mathbf{5}$ (155.3 $\mathrm{mg}, 537 \mu \mathrm{mol})$ in anhydrous DMF $(2 \mathrm{~mL})$ and $\mathrm{Et}_{3} \mathrm{~N}(0.100 \mathrm{~mL})$ by blowing argon for $20 \mathrm{~min}$. Then $(o \text {-tol })_{3} \mathrm{P}(14.2 \mathrm{mg}, 46.7 \mu \mathrm{mol})$ and $\mathrm{Pd}(\mathrm{OAc})_{2}(5.7 \mathrm{mg}, 23.4 \mu \mathrm{mol})$ were added. The reaction mixture was heated for $7 \mathrm{~h}$ at $100^{\circ} \mathrm{C}$. After evaporation of the solvents, the crude product was purified by column chromatography (heptane $/ \mathrm{CH}_{2} \mathrm{Cl}_{2} 20: 80$ ) to yield $122 \mathrm{mg}$ $(70 \%)$ of $6 \mathrm{a} ;{ }^{1} \mathrm{H}$ NMR $\left(200.13 \mathrm{MHz}, \mathrm{CDCl}_{3}\right) \delta 9.92(\mathrm{~s}, 1 \mathrm{H}), 8.05$ and $7.79\left(\mathrm{AA}^{\prime} \mathrm{XX}, J_{\mathrm{AX}}=\right.$ $8.4 \mathrm{~Hz}, 8 \mathrm{H}), 7.81$ and $7.24\left(\mathrm{AA}^{\prime} \mathrm{XX}{ }^{\prime}, J_{\mathrm{AX}}=8.3 \mathrm{~Hz}, 4 \mathrm{H}\right), 7.59$ and $7.24\left(\mathrm{AA}^{\prime} \mathrm{XX}{ }^{\prime}, J_{\mathrm{AX}}=8.5\right.$ $\mathrm{Hz}, 8 \mathrm{H}), 7.37$ (d, $J=16.3 \mathrm{~Hz}, 2 \mathrm{H}), 7.15(\mathrm{~d}, J=16.3 \mathrm{~Hz}, 2 \mathrm{H}) ;{ }^{13} \mathrm{C}$ NMR $\left(50.32 \mathrm{MHz}, \mathrm{CDCl}_{3}\right)$ $\delta 190.5,152.3,146.7,145.7,133.4,132.5,131.3,130.6,128.8,128.6,127.3,125.7,125.6$, $123.4,121.7,119.8$ (q, $J=325.8 \mathrm{~Hz}$ ); HRMS $\left(\mathrm{ES}^{+}, \mathrm{MeOH}\right)$ calcd for $\mathrm{C}_{37} \mathrm{H}_{25} \mathrm{NO}_{5} \mathrm{~F}_{6} \mathrm{NaS}_{2}$ $\left([\mathrm{M}+\mathrm{Na}]^{+}\right) \mathrm{m} / \mathrm{z}$ 764.0976, found 764.0979.

(4-Ethenylphenyl)bis[4-[(1E)-2-[4-[(trifluoromethyl)sulfonyl]phenyl]-ethenyl]phenyl]amine (6b). To a solution of aldehyde $\mathbf{6 a}(74.3 \mathrm{mg}, 100 \mu \mathrm{mol})$ and methyltriphenylphosphonium iodide $(81 \mathrm{mg}, 200 \mu \mathrm{mol})$ in anhydrous THF $(7.5 \mathrm{~mL})$ was 
added sodium hydride $(8.2 \mathrm{mg}, 60 \%$ dispersion in mineral oil). The reaction mixture was stirred for $4 \mathrm{~h}$ at $20^{\circ} \mathrm{C}$. After filtration through Celite, the crude product was purified by column chromatography (heptane $/ \mathrm{CH}_{2} \mathrm{Cl}_{2} 20: 80$ ) to yield $40.3 \mathrm{mg}(54 \%)$ of $\mathbf{6 b} ;{ }^{1} \mathrm{H} \mathrm{NMR}$ $\left(200.13 \mathrm{MHz}, \mathrm{CDCl}_{3}\right) \delta 8.04$ and $7.78\left(\mathrm{AA}^{\prime} \mathrm{XX}\right.$ ', $\left.J_{\mathrm{AX}}=8.3 \mathrm{~Hz}, 8 \mathrm{H}\right), 7.51$ and $7.18\left(\mathrm{AA}^{\prime} \mathrm{XX}\right.$ ', $\left.J_{\mathrm{AX}}=8.6 \mathrm{~Hz}, 8 \mathrm{H}\right), 7.42$ and $7.16\left(\mathrm{AA}^{\prime} \mathrm{XX} X^{\prime}, J_{\mathrm{AX}}=8.6 \mathrm{~Hz}, 4 \mathrm{H}\right), 7.35(\mathrm{~d}, J=16.1 \mathrm{~Hz}, 2 \mathrm{H}), 7.10$ $(\mathrm{d}, J=16.1 \mathrm{~Hz}, 2 \mathrm{H}), 6.75(\mathrm{dd}, J=17.4 \mathrm{~Hz}, J=11.0 \mathrm{~Hz}, 1 \mathrm{H}), 5.76(\mathrm{~d}, J=17.4 \mathrm{~Hz}, 1 \mathrm{H}), 5.33$ $(\mathrm{d}, J=11.0 \mathrm{~Hz}, 1 \mathrm{H}) ;{ }^{13} \mathrm{C} \mathrm{NMR}\left(75.47 \mathrm{MHz}, \mathrm{CDCl}_{3}\right) \delta 146.8,145.1,145.0,134.9,132.7$, 132.6, 130.2, 129.6, 127.5, 127.3, 126.4, 126.1, 124.2, 123.4, 122.8, 118.8 (q, $J=326.0 \mathrm{~Hz}$ ), 112.3; HRMS $\left(\mathrm{ES}^{+}, \mathrm{MeOH}\right)$ calcd for $\mathrm{C}_{38} \mathrm{H}_{27} \mathrm{NO}_{4} \mathrm{~F}_{6} \mathrm{~S}_{2} \mathrm{~K}\left([\mathrm{M}+\mathrm{K}]^{+}\right) \mathrm{m} / z$ 778.0923, found 778.0891 .

Tris[4-[(1E)-2-[4-[(trifluoromethyl)sulfonyl]phenyl]ethenyl]phenyl]amine (O1). Air was removed from a solution of $\mathbf{4 b}(60 \mathrm{mg}, 185 \mu \mathrm{mol})$ and $\mathbf{5}(187 \mathrm{mg}, 649 \mu \mathrm{mol})$ in anhydrous DMF $(1 \mathrm{~mL})$ and $\mathrm{Et}_{3} \mathrm{~N}(0.100 \mathrm{~mL})$ by blowing argon for $20 \mathrm{~min}$. Then $(o \text {-tol })_{3} \mathrm{P}(17 \mathrm{mg}, 55$ $\mu \mathrm{mol})$ and $\mathrm{Pd}(\mathrm{OAc})_{2}(7 \mathrm{mg}, 28 \mu \mathrm{mol})$ were added. The reaction mixture was heated for $3 \mathrm{~h}$ at $100^{\circ} \mathrm{C}$. After evaporation of the solvents, the crude product was purified by column chromatography (heptane $/ \mathrm{CH}_{2} \mathrm{Cl}_{2}$ 50:50) to yield $133 \mathrm{mg}(75 \%)$ of $\mathbf{O 1} ; \mathrm{mp} 135-136{ }^{\circ} \mathrm{C} ;{ }^{1} \mathrm{H}$ NMR (300.13 MHz, $\left.\mathrm{CDCl}_{3}\right) \delta 8.00$ and $7.74\left(\mathrm{AA}^{\prime} \mathrm{XX}{ }^{\prime}, J_{\mathrm{AX}}=8.5 \mathrm{~Hz}, 12 \mathrm{H}\right), 7.52$ and 7.17 $\left(\mathrm{AA}^{\prime} \mathrm{XX}, J_{\mathrm{AX}}=8.5 \mathrm{~Hz}, 12 \mathrm{H}\right), 7.33(\mathrm{~d}, J=16.5 \mathrm{~Hz}, 3 \mathrm{H}), 7.09(\mathrm{~d}, J=16.5 \mathrm{~Hz}, 3 \mathrm{H}) ;{ }^{13} \mathrm{C} \mathrm{NMR}$ $\left(75.47 \mathrm{MHz}, \mathrm{CDCl}_{3}\right) \delta 147.5,145.8,133.6,131.2,128.7,128.4,127.1,124.9,124.5,119.9$ (q, $J=325.9 \mathrm{~Hz}) ;{ }^{19} \mathrm{~F}$ NMR $\left(282.37 \mathrm{MHz}, \mathrm{CDCl}_{3}\right): \delta$-78.42; HRMS (LSIMS ${ }^{+}$, mNBA) calcd for $\mathrm{C}_{45} \mathrm{H}_{30} \mathrm{~F}_{9} \mathrm{NO}_{6} \mathrm{~S}_{3}\left(\mathrm{M}^{+} \cdot\right) \mathrm{m} / z$ 947.1092, found 947.1105. Anal. Calcd for $\mathrm{C}_{45} \mathrm{H}_{30} \mathrm{~F}_{9} \mathrm{NO}_{6} \mathrm{~S}_{3}$ (947.93): C, 57.02; H, 3.19; N, 1.48. Found: C, 56.63; H, 3.47; N, 1.31.

\section{2-[[4-[(1E)-2-[4-[Bis[4-[(1E)-2-[4-[(trifluoromethyl)sulfonyl]phenyl]-ethenyl]phenyl]-} aminolphenyl]ethenyl]phenyl]sulfonyl]ethanol (O2). Air was removed from a solution of 6b $(39.5 \mathrm{mg}, 53.4 \mu \mathrm{mol})$ and $7(18.4 \mathrm{mg}, 69.4 \mu \mathrm{mol})$ in anhydrous DMF (1 mL) and $\mathrm{Et}_{3} \mathrm{~N}(40$ $\mu \mathrm{L})$ by blowing argon for $20 \mathrm{~min}$. Then $(o-\text { tol })_{3} \mathrm{P}(1.6 \mathrm{mg}, 5.3 \mu \mathrm{mol})$ and $\mathrm{Pd}(\mathrm{OAc})_{2}(0.6 \mathrm{mg}$, $2.7 \mu \mathrm{mol})$ were added. The reaction mixture was heated for $21 \mathrm{~h}$ at $100^{\circ} \mathrm{C}$. After evaporation of the solvents, the crude product was purified by column chromatography $\left(\mathrm{CH}_{2} \mathrm{Cl}_{2} / \mathrm{EtOAc}\right.$, $90: 10)$ to yield $26.1 \mathrm{mg}(53 \%)$ of $\mathbf{O 2} ; \mathrm{mp} 116^{\circ} \mathrm{C} ;{ }^{1} \mathrm{H}$ NMR $\left(200.13 \mathrm{MHz}, \mathrm{CDCl}_{3}\right) \delta 8.00$ and $7.74\left(\mathrm{AA}^{\prime} \mathrm{XX} X^{\prime}, J_{\mathrm{AX}}=8.5 \mathrm{~Hz}, 8 \mathrm{H}\right), 7.91$ and $7.69\left(\mathrm{AA}^{\prime} \mathrm{XX}^{\prime}, J_{\mathrm{AX}}=8.4 \mathrm{~Hz}, 4 \mathrm{H}\right), 7.50$ and 7.16 $\left(\mathrm{AA}^{\prime} \mathrm{XX}\right.$ ', $\left.J_{\mathrm{AX}}=8.5 \mathrm{~Hz}, 12 \mathrm{H}\right), 7.31(\mathrm{~d}, J=16.6 \mathrm{~Hz}, 3 \mathrm{H}), 7.07(\mathrm{~d}, J=16.6 \mathrm{~Hz}, 3 \mathrm{H}), 4.04(\mathrm{dt}, J$ $=5.9 \mathrm{~Hz}, 5.3 \mathrm{~Hz}, 2 \mathrm{H}), 3.38(\mathrm{t}, J=5.3 \mathrm{~Hz}, 2 \mathrm{H}), 2.77(\mathrm{t}, J=5.9 \mathrm{~Hz}, 1 \mathrm{H}) ;{ }^{13} \mathrm{C} \mathrm{NMR}(75.47$ $\left.\mathrm{MHz}, \mathrm{CDCl}_{3}\right) \delta 147.6,147.1,145.9,143.3,136.9,133.6,132.1,131.7,131.3,131.1,128.5$, $128.4,128.2$, 127.2, 127.2, 127.0, 125.4, 124.8, 124.7, $124.3,119.9$ (q, $J=325.8 \mathrm{~Hz}$ ), 58.3, 56.5; ${ }^{19} \mathrm{~F}$ NMR $\left(282.37 \mathrm{MHz}, \mathrm{CDCl}_{3}\right) \delta-78.43$; HRMS $\left(\mathrm{ES}^{+}, \mathrm{MeOH}\right)$ calcd for $\mathrm{C}_{46} \mathrm{H}_{35} \mathrm{~F}_{6} \mathrm{NO}_{7} \mathrm{~S}_{3} \mathrm{Na}\left([\mathrm{M}+\mathrm{Na}]^{+}\right) \mathrm{m} / z$ 946.1378, found 946.1384 .

Tris[4-[(1E)-2-[4-[(2-hydroxyethyl)sulfonyl]phenyl]ethenyl]phenyl]amine (O3). Air was removed from a solution of $\mathbf{4 b}(70 \mathrm{mg}, 0.216 \mathrm{mmol})$ and $7(200 \mathrm{mg}, 0.758 \mathrm{mmol})$ in anhydrous DMF $(2 \mathrm{~mL})$ and $\mathrm{Et}_{3} \mathrm{~N}(0.120 \mathrm{~mL})$ by blowing argon for $20 \mathrm{~min}$. Then $(o \text {-tol })_{3} \mathrm{P}$ $(19.8 \mathrm{mg}, 65 \mu \mathrm{mol})$ and $\mathrm{Pd}(\mathrm{OAc})_{2}(7.3 \mathrm{mg}, 32.5 \mu \mathrm{mol})$ were added. The reaction mixture was 
heated for $3 \mathrm{~h}$ at $100^{\circ} \mathrm{C}$. After evaporation of the solvents, the crude product was purified by column chromatography $\left(\mathrm{CH}_{2} \mathrm{Cl}_{2} / \mathrm{AcOEt}\right.$, gradient from $95: 5$ to $50: 50$, then $\mathrm{CH}_{2} \mathrm{Cl}_{2} / \mathrm{EtOH}$ 90 : 5) to yield $79 \mathrm{mg}(42 \%)$ of $\mathbf{O 3}$; mp $155-156{ }^{\circ} \mathrm{C} ;{ }^{1} \mathrm{H}$ NMR $\left(200.13 \mathrm{MHz}, \mathrm{CDCl}_{3}\right) \delta 7.90$ and $7.68\left(\mathrm{AA}^{\prime} \mathrm{XX}\right.$ ', $\left.J_{\mathrm{AX}}=8.4 \mathrm{~Hz}, 12 \mathrm{H}\right), 7.48$ and $7.15\left(\mathrm{AA}^{\prime} \mathrm{XX}\right.$ ', $\left.J_{\mathrm{AX}}=8.6 \mathrm{~Hz}, 12 \mathrm{H}\right), 7.24(\mathrm{~d}$, $J=16.2 \mathrm{~Hz}, 3 \mathrm{H}), 7.05(\mathrm{~d}, J=16.2 \mathrm{~Hz}, 3 \mathrm{H}), 4.03(\mathrm{~m}, 6 \mathrm{H}), 3.38(\mathrm{t}, J=5.3 \mathrm{~Hz}, 6 \mathrm{H}), 2.80(\mathrm{~s}$, $3 \mathrm{H}) ;{ }^{13} \mathrm{C} \mathrm{NMR}\left(50.32 \mathrm{MHz}, \mathrm{CDCl}_{3}\right) \delta 147.3,143.4,136.8,132.1,131.5,128.5,128.2,127.0$, 125.3, 124.2, 58.4, 56.5; HRMS $\left(\mathrm{ES}^{+}, \mathrm{MeOH}\right)$ calcd for $\mathrm{C}_{48} \mathrm{H}_{45} \mathrm{NO}_{9} \mathrm{~S}_{3} \mathrm{Na}\left([\mathrm{M}+\mathrm{Na}]^{+}\right) \mathrm{m} / \mathrm{z}$ 898.2154 , found 898.2150 .

\section{Optical absorption and emission spectroscopy}

All photophysical properties have been performed with freshly-prepared air-equilibrated solutions at room temperature $(298 \mathrm{~K})$. UV/Vis absorption spectra were recorded on a Jasco V-570 spectrophotometer. Steady-state and time-resolved fluorescence measurements were performed on dilute solutions $\left(\mathrm{ca} \cdot 10^{-6} \mathrm{M}\right.$, optical density $\left.<0.1\right)$ contained in standard $1 \mathrm{~cm}$ quartz cuvettes using an Edinburgh Instruments (FLS920) spectrometer in photon-counting mode. Emission spectra were obtained, for each compound, under excitation at the wavelength of the absorption maximum. Fluorescence quantum yields were measured according to literature procedures using fluorescein in $0.1 \mathrm{~N} \mathrm{NaOH}$ as a standard (quantum yield $\Phi=0.90) .{ }^{82-83}$ The lifetime values were obtained from the reconvolution fit analysis (Edinburgh F900 analysis software) of decay profiles obtained using the FLS920 instrument under excitation with a nitrogen-filled nanosecond flashlamp. The quality of the fits was evidenced by the reduced $\chi^{2}$ value $\left(\chi^{2}<1.1\right)$.

\section{Two-photon excitation}

To span the 700-980 nm range, a Nd:YLF-pumped Ti:sapphire oscillator was used generating $150 \mathrm{fs}$ pulses at a $76 \mathrm{MHz}$ rate. The excitation was focused into the cuvette through a microscope objective (10x, NA 0.25). The fluorescence was detected in epifluorescence mode via a dichroic mirror (Chroma 675dcxru) and a barrier filter (Chroma e650sp-2p) by a compact CCD spectrometer module BWTek BTC112E. Total fluorescence intensities were obtained by integrating the corrected emission spectra measured by this spectrometer. TPA cross-sections $\left(\sigma_{2}\right)$ were determined from the two-photon excited fluorescence (TPEF) crosssections $\left(\sigma_{2} \Phi\right)$ and the fluorescence emission quantum yield $(\Phi)$. TPEF cross-sections of $10^{-4}$ $\mathrm{M}$ solutions (toluene for quadrupoles and ethanol for octupoles were chosen for solubility reasons) were measured relative to fluorescein in $0.01 \mathrm{M}$ aqueous $\mathrm{NaOH}$ for $715-980 \mathrm{~nm},{ }^{84-85}$ using the well-established method described by $\mathrm{Xu}$ and $\mathrm{Webb}^{84}$ and the appropriate solventrelated refractive index corrections. ${ }^{86}$ Data points between 700 and $715 \mathrm{~nm}$ were corrected according to Ref. ${ }^{81}$ The quadratic dependence of the fluorescence intensity on the excitation power was checked for each sample and all wavelengths, indicating that the measurements were carried out in intensity regimes where saturation or photodegradation did not occur. 


\section{Electrochemical experiments}

Voltammetric experiments with the scan rates ranging from $v=0.01$ to $0.5 \mathrm{~V} \mathrm{~s}^{-1}$ were performed using an EG\&G Model 362 potentiostat in three electrodes mode and tracing voltammograms with a Kipp and Zonen flatbed recorder. For higher scan rates, an Autolab PGSTAT100 was employed using its IR compensating facility. Given relatively high adsorptive ability of the compounds studied along with their very low solubility in $\mathrm{CH}_{3} \mathrm{CN}$, several electrodes were used for voltammetric experiments: $0.2 \mathrm{~mm}$ or $3 \mathrm{~mm}$ diameter glassy carbon (GC) electrodes, $300 \mu \mathrm{m}$ Pt and $0.5 \mathrm{~mm}$ Au microdiscs. Au anode was more suitable in less polar binary mixtures $\mathrm{CH}_{3} \mathrm{CN}-\mathrm{THF}$, glassy carbon electrode allowed better voltammograms in more polar media and the $\mathrm{Pt}$ electrode provided the less reproducible results. Auxiliary electrode was a $\varnothing 2.5 \times 50 \mathrm{~mm}$ GC rod separated from the analyte by an electrolytic bridge filled with a $0.1 \mathrm{M} \mathrm{Bu}_{4} \mathrm{NPF}_{6}$ solution in $\mathrm{THF} / \mathrm{CH}_{3} \mathrm{CN}(4: 1 \mathrm{v} / \mathrm{v})$. The working electrodes were carefully polished with Struers FEPA P 4000 paper and rinsed consecutively with acetonitrile and diethyl ether before each run. All reported potentials were measured versus $\mathrm{Ag} / 0.1 \mathrm{M} \mathrm{AgNO}_{3}$ in $\mathrm{CH}_{3} \mathrm{CN}$ reference electrode and $\mathrm{Fc}^{+} / \mathrm{Fc}$ reversible couple was used as a standard allowing to have an internal reference for proper IRcompensation and to correct the measured potentials for liquid junction potential when the solubility of the compounds required different $\mathrm{THF}-\mathrm{CH}_{3} \mathrm{CN}$ ratio. EPR spectra were obtained using a Bruker EMX X-band spectrometer operating at the frequency $9.46 \mathrm{GHz}$.

\section{Essential-state approach}

Essential-state models have been used in the field of NLO and TPA for gaining insight in the NLO responses (including solvent effects) of different chromophores (including push-pull, ${ }^{87-}$ ${ }^{92}$ quadrupolar, ${ }^{55,67,93}$ octupolar ${ }^{36,57,94}$ but also porphyrins and phtalocyanins derivatives).$^{74,95}$

The essential-state model for quadrupolar chromophores accounts for three basis states corresponding to the neutral structure, $|N>=| \mathrm{DAD}>$, and to two degenerate structures, $\left|Z_{1}\right\rangle$ $=\mid \mathrm{D}^{+} \mathrm{A}-\mathrm{D}>$ and $\left|Z_{2}\right\rangle=\mid \mathrm{DA} \cdot \mathrm{D}^{+}>$(of course analogous structures can be written for ADA chromophores). ${ }^{55,67}$ The two charge-separated states $Z_{1}$ and $Z_{2}$ are degenerate and are separated by an energy gap $2 z$ from the neutral state. A matrix element $-\sqrt{2} t$ mixes $N$ to $Z_{1}$ and $Z_{2}$, whereas the direct mixing between $Z_{1}$ and $Z_{2}$ is neglected. Exploiting inversion symmetry, we can combine the two zwitterionic states in phase and out of phase: $Z_{ \pm}=\left(Z_{1} \pm Z_{2}\right) / \sqrt{2}$. The antisymmetric state, $Z$, stays unmixed and corresponds to the lowest excited state, $c$, allowed in one-photon absorption processes. The two symmetric states, $N$ and $Z_{+}$mix into a ground and an excited state, $g=\sqrt{1-\rho}|N\rangle+\sqrt{\rho}\left|Z_{+}\right\rangle$and $e=\sqrt{\rho}|N\rangle-\sqrt{1-\rho}\left|Z_{+}\right\rangle$, where $\rho$ measures the weight of the $Z_{+}$state into the ground state or, equivalently, the average charge on the central site, and is therefore proportional to the quadrupolar molecular moment. We define $\mu_{0}$ as the dipole moment of the charge-separated $Z_{1}$ state (an equal and opposite dipole moment is of course assigned to $Z_{2}$ ), so that all quantities of spectroscopic interest may be expressed as a function of $\rho .^{55}$

Octupolar chromophores of $\mathrm{DA}_{3}$ (or $\mathrm{AD}_{3}$ ) structure can be described as resonating between a neutral form, $|N\rangle$ and three charge-separated states $\left|Z_{1}\right\rangle,\left|Z_{2}\right\rangle$ and $\left|Z_{3}\right\rangle$, corresponding to the three degenerate states $\mathrm{D}^{+} \mathrm{A}-\mathrm{A}_{2}$ (or $\mathrm{A}^{-} \mathrm{D}^{+} \mathrm{D}_{2}$ ). ${ }^{57,94}$ The three charge- 
separated states have dipole moments of equal length, $\mu_{0}$, pointing along the three molecular arms. As before, $2 z$ is the energy gap between the charge-separated states and the neutral state, while $-\sqrt{2} t$ is the matrix element that mixes $|N\rangle$ to each one of the three $\left|Z_{i}\right\rangle$ states. The three arms of the molecule are interchanged by a $C_{3}$ axis: the three degenerate basis states are conveniently combined into a symmetric wavefunction: $Z_{+}=\left(Z_{1}+Z_{2}+Z_{3}\right) / \sqrt{3}$ and two $E$-symmetry functions that may be chosen as: $c_{1}=\left(2 Z_{1}-Z_{2}-Z_{3}\right) / \sqrt{6}$ and $c_{2}=\left(Z_{2}-Z_{3}\right) / \sqrt{2}$. The neutral wavefunction $N$ has the same symmetry as $Z_{+}$: the two states are mixed to give two totally-symmetric states, the ground state, $g$, and an excited state, $e$, that are described by the same expressions as reported for quadrupolar dyes, with $\rho$ measuring the average charge on the central site, proportional to the octupolar molecular moment. The $E$ symmetry states stay unmixed and correspond to two degenerate excited states, $c_{1}$ and $c_{2}$, accessible by OPA. At variance with quadrupolar dyes, all excited states can be reached by TPA, even if the transition to the $e$-state largely dominates the TPA spectrum. ${ }^{57}$

The electronic essential-state models for quadrupolar and octupolar chromophores can be extended to account for the coupling between electronic and vibrational degrees of freedom and for polar solvation. These models proved fairly successful in the description of linear and nonlinear spectra of several chromophores. ${ }^{57,94}$ Polar solvation can be accounted for describing the solvent as a continuum dielectric medium. ${ }^{96}$ The polar molecules of the solvent reorient around the solute to generate a reaction field, $F_{o r}$, proportional to the solute dipole moment. The reaction field enters the molecular Hamiltonian adding an interaction term $-\mu F_{o r}$ plus an elastic restoring energy $\mu_{0}^{2} F_{o r}{ }^{2} /\left(4 \varepsilon_{o r}\right)$, where the solvation relaxation energy, $\varepsilon_{o r}$, increases with the solvent polarity. The orientational motion is very slow and can be treated classically. ${ }^{57}$ In this approximation each solute molecule experiences a slightly different local reaction field. The molecular Hamiltonian is then diagonalized for different $F_{o r}$, and spectra are calculated as sums of contributions from the different molecules, weighted according to the Boltzmann distribution on the energies.

The model for molecular vibrations needs at least one degree of freedom for each molecular arm. In quadrupolar chromophores, two effective vibrational coordinates describe the independent vibrations of the two molecular arms. The two coordinates are conveniently combined into a symmetric and an antisymmetric vibration. ${ }^{55}$ Specifically, each basis states is assigned a harmonic potential energy surfaces (PES) with the same frequency, $\omega_{v}$, but with displaced minima along the relevant vibrational coordinate, to describe the different equilibrium geometry for each state. The strength of the coupling is conveniently measured by $\varepsilon_{s p}$, the vibrational relaxation energy associated with the $|N>\rightarrow| Z_{i}>$ process. Similarly, for octupolar chromophores three effective molecular coordinates enter the model, that combine to give a symmetric coordinate and two degenerate $E$-symmetry coordinates. ${ }^{57}$ The presence of non-totally symmetric vibrations in quadrupolar and octupolar chromophores leads to interesting spectroscopic effects, such as vibronic activation (through the so-called HerzbergTeller mechanism) of forbidden transitions, and symmetry-breaking or localization phenomena, whose manifestation has been recently recognized in the largely solvatochromic fluorescence observed for quadrupolar and octupolar dyes. ${ }^{55-57}$ 
Parameters fixed in order to reproduce spectral properties of the studied compounds are specified in the captions of Figures 5 and 6 . The different donor/acceptor strengths between purely quadrupolar chromophores $\mathbf{Q 1}$ and $\mathbf{Q 3}$ and between purely octupolar compounds $\mathbf{O 1}$ and $\mathbf{O 3}$ were modeled by simply assigning a different $2 z$ value. Dissymmetrical quadrupolar and octupolar chromophores have been modeled along the same lines, but assigning different energies $\left(2 z_{1}\right.$ and $\left.2 z_{2}\right)$ to the zwitterionic basis states corresponding to charge separation on different molecular branches. All other parameters were kept fixed.

Numerically exact nonadiabatic diagonalizations of the coupled electron-phonon problems have been performed, avoiding the problematic calculation of the dynamics on adiabatic potential energy surfaces. The nonadiabatic basis is obtained as the direct product of the electronic basis times the eigenstates of the harmonic oscillators associated with the effective molecular coordinates. The eigenstates of each harmonic oscillator define an infinite basis, which is truncated by fixing a maximum number of phonon states, $M$, for each oscillator, to give a truncated basis with dimension $3 \mathrm{M}^{2}$ for quadrupoles, and $4 \mathrm{M}^{3}$ for octupoles. The corresponding Hamiltonian matrices can be diagonalized up to fairly large $\mathrm{M}$ values to yield numerically exact nonadiabatic eigenstates. The minimum $\mathrm{M}$ required for convergence depends on the model parameters and on the properties of interest. All results presented here were obtained with $M=10$ for quadrupoles, $M=6$ for octupoles.

\section{Quantum chemistry modeling}

For the sake of simplicity, nonyl and hexyl solubilizing chains have been replaced by methyl groups. Solvation effects were neglected. ${ }^{2}$ Ground-state optimized geometries have been obtained using the Gaussian 03 package. ${ }^{97}$ For ground state geometries, we previously found that the Hartree-Fock (HF) method is superior to the DFT-based approaches by reproducing accurately bond length alternation parameter in similar conjugated systems when compared to experiment. ${ }^{40}$ Thus, all ground-state geometries are obtained at the HF level using the 6-31G basis set. We note that using DFT-based kernels (such as B3LYP) for ground-state geometry optimizations would lead to consistent red-shifts in the excitation energies and slightly increased OPA and TPA response amplitudes compared to respective quantities, calculated at HF level. HOMO-LUMO gaps have been calculated at B3LYP/6$31 \mathrm{G}+$ level of theory.

Hybrid functionals such as B3LYP have been found to be very accurate for excited states in many molecular systems. ${ }^{40,98-100}$ Consequently, we used TD-B3LYP/6-31G level of theory to investigate linear and nonlinear optical properties using the density matrix formalism for nonlinear optical responses as described in ref. ${ }^{101}$ This method has already demonstrated its relevance ${ }^{2,40,81}$ for both linear and nonlinear absorption spectra in different series of substituted chromophores. Excited-state electronic structures, with 20 excited states for quadrupolar compounds Q1-Q3 and 60 for octupolar compound O1-O3, were calculated with Gaussian 03. ${ }^{97}$ Vertical transition frequencies, dipoles and densities are used to model both linear absorption and TPA spectra. ${ }^{101}$ In particular, the third order polarizability $\gamma(-\omega ; \omega$; $\omega ;-\omega)$ has been calculated using the density-matrix formalism as described in ref. ${ }^{101}$ 
following Eq. (6) in ref. ${ }^{102}$ The TPA cross-section $\sigma_{2}(\omega)$ in vacuum is related to the imaginary part of the orientational average of $\gamma(-\omega ; \omega ; \omega ;-\omega)$ :

$\langle\gamma\rangle=\frac{1}{15}\left(3 \sum_{i} \gamma_{i i i i}+\sum_{j \neq i}\left(\gamma_{i i j j}+\gamma_{i j i j}+\gamma_{i j j i}\right)\right)$,

by

$\sigma_{2}(\omega)=\frac{24 \pi^{2} \hbar \omega^{2}}{c^{2}} \operatorname{Im}\langle\gamma\rangle$,

where $\hbar$ is Planck's constant and $c$ is the speed of light. The damping factor introduced to simulate finite linewidths in the resonant spectra has been fixed to $0.17 \mathrm{eV}$ for all chromophores (Tab. S1). All comparisons with the experimental response amplitudes are subject to uncertainties related to the choice of the line-broadening parameter and to consideration of solvation effects. ${ }^{2}$

To model fluorescence, we used the TURBOMOLE ${ }^{103}$ package to optimize molecular geometries of the lowest singlet excited state at the TD-HF/SV level.

Natural transition orbital analysis of the excited states ${ }^{104}$ has been used to analyze the nature of the excited states involved in the photophysical processes. They offer the most compact representation of a given transition density in terms of an expansion into single-particle transitions. Figures showing molecular geometries and transition orbitals were obtained with XcrySDen. ${ }^{105}$

\section{Acknowledgement}

MBD and ST gratefully acknowledge CNRS for an invited research associate position to ST. MBD, CK and,FT acknowledge the Italo-French University and Egide for funding through the Galileo Project. This work was performed in part at the US Department of Energy, Center for Integrated Nanotechnologies (CINT), at Los Alamos National Laboratory (LANL) (Contract DE-AC52-06NA25396). MBD acknowledges financial support from DGA (Grant 00.34.070.00.470.75.653) and Rennes Métropole (equipment grant "Allocation Spécifique d'Installation"). MC received fellowship from DGA. We acknowledge support of Center for Nonlinear Studies (CNLS) at LANL. We thank E. Leroux for technical assistance in the synthesis, S. Soualmi in electrochemical mesaurements and M. H. V. Werts for help in the TPEF measurements. FT acknowledges MIUR for funding through PRIN2006-031511. A portion of the calculations was funded by the "Centre Informatique National de l'Enseignement Supérieur" (CINES-France).

Supporting information available: Additional calculated and experimental absorption spectra and table. This material is available free of charge via the Internet at http:/pubs.acs.org. 


\section{References}

(1) He, G. S.; Tan, L.-S.; Zheng, Q.; Prasad, P. N. Chem. Rev. 2008, 108, 12451330.

(2) Terenziani, F.; Katan, C.; Badaeva, E.; Tretiak, S.; Blanchard-Desce, M. Adv. Mater. 2008, 20, 4641-4678.

(3) Pawlicki, M.; Collins, H. A.; Denning, R. G.; Anderson, H. L. Angew. Chem., Int. Ed. 2009, 48, 3244-3266.

(4) Kim, H. M.; Cho, B. R. Chem. Commun. 2009, 153-164.

(5) Denk, W.; Strickler, J. H.; Webb, W. W. Science 1990, 248, 73-76.

(6) Kim, H. M.; Cho, B. R. Acc. Chem. Res. 2009, 42, 863-872.

(7) Bhawalkar, J. D.; Kumar, N. D.; Zhao, C. F.; Prasad, P. N. J. Clin. Laser Med. Surg. 1997, 15, 201-204.

(8) Collins, H. A.; Khurana, M.; Moriyama, E. H.; Mariampillai, A.; Dahlstedt, E.; Balaz, M.; Kuimova, M. K.; Drobizhev, M.; Yang, V. X. D.; Phillips, D.; Rebane, A.; Wilson, B. C.; Anderson, H. L. Nat. Photonics 2008, 2, 420-424.

(9) Parthenopoulos, D. A.; Rentzepis, P. M. Science 1989, 245, 843-845.

(10) Kawata, S.; Sun, H.-B.; Tanaka, T.; Takada, K. Nature 2001, 412, 697-698.

(11) He, G. S.; Xu, G. C.; Prasad, P. N.; Reinhardt, B. A.; Bhatt, J. C.; McKellar, R.; Dillard, A. G. Opt. Lett. 1995, 20, 435-437.

(12) Prasad, P. N. Introduction to Biophotonics; John Wiley and Sons: New York, 2003.

(13) Robin, A.-C.; Gmouh, S.; Mongin, O.; Jouikov, V.; Werts, M. H. V.; Gautier, C.; Slama-Schwok, A.; Blanchard-Desce, M. Chem. Commun. 2007, 1334-1336.

(14) Larson, D. R.; Zipfel, W. R.; Williams, R. M.; Clark, S. W.; Bruchez, M. P.; Wise, F. W.; Webb, W. W. Science 2003, 300, 1434-1437.

(15) Krishna, T. R.; Parent, M.; Werts, M. H. V.; Moreaux, L.; Gmouh, S.; Charpak, S.; Caminade, A.-M.; Majoral, J.-P.; Blanchard-Desce, M. Angew. Chem., Int. Ed. 2006, 45, 4645-4648.

165.

(16) Svoboda, K.; Denk, W.; Kleinfeld, D.; Tank, D. W. Nature 1997, 385, 161-

(17) Zipfel, W. R.; Williams, R. M.; Christie, R.; Nikitin, A. Y.; Hyman, B. T.; Webb, W. W. Proc. Natl. Acad. Sci. U. S. A. 2003, 100, 7075-7080.

(18) He, G. S.; Bhawalkar, J. D.; Zhao, C. F.; Prasad, P. N. Appl. Phys. Lett. 1995, $67,2433-2435$.

(19) Abbotto, A.; Beverina, L.; Bozio, R.; Bradamante, S.; Ferrante, C.; Pagani, G. A.; Signorini, R. Adv. Mater. 2000, 12, 1963-1967.

(20) Kannan, R.; He, G. S.; Yuan, L.; Xu, F.; Prasad, P. N.; Dombroskie, A. G.; Reinhardt, B. A.; Baur, J. W.; Vaia, R. A.; Tan, L.-S. Chem. Mater. 2001, 13, 1896-1904.

(21) Charier, S.; Ruel, O.; Baudin, J.-B.; Alcor, D.; Allemand, J.-F.; Meglio, A.; Jullien, L. Angew. Chem., Int. Ed. 2004, 43, 4785-4788.

(22) Reinhardt, B. A.; Brott, L. L.; Clarson, S. J.; Dillard, A. G.; Bhatt, J. C.; Kannan, R.; Yuan, L.; He, G. S.; Prasad, P. N. Chem. Mater. 1998, 10, 1863-1874.

(23) Albota, M.; Beljonne, D.; Brédas, J.-L.; Ehrlich, J. E.; Fu, J.-Y.; Heikal, A. A.; Hess, S. E.; Kogej, T.; Levin, M. D.; Marder, S. R.; McCord-Maughon, D.; Perry, J. W.; Röckel, H.; Rumi, M.; Subramaniam, G.; Webb, W. W.; Wu, X.-L.; Xu, C. Science 1998, 281, 1653-1656.

(24) Ventelon, L.; Moreaux, L.; Mertz, J.; Blanchard-Desce, M. Chem. Commun. 1999, 2055-2056. 
(25) Ventelon, L.; Charier, S.; Moreaux, L.; Mertz, J.; Blanchard-Desce, M. Angew. Chem., Int. Ed. 2001, 40, 2098-2101.

(26) Frederiksen, P. K.; Jørgensen, M.; Ogilby, P. R. J. Am. Chem. Soc. 2001, 123, 1215-1221.

(27) Mongin, O.; Porrès, L.; Moreaux, L.; Mertz, J.; Blanchard-Desce, M. Org. Lett. 2002, 4, 719-722.

(28) Abbotto, A.; Beverina, L.; Bozio, R.; Facchetti, A.; Ferrante, C.; Pagani, G. A.; Pedron, D.; Signorini, R. Org. Lett. 2002, 4, 1495-1498.

(29) Yang, W. J.; Kim, D. Y.; Jeong, M.-Y.; Kim, H. M.; Jeon, S.-J.; Cho, B. R. Chem. Commun. 2003, 2618-2619.

(30) Strehmel, B.; Sarker, A. M.; Detert, H. ChemPhysChem 2003, 4, 249-259.

(31) Lee, S. K.; Yang, W. J.; Choi, J. J.; Kim, C. H.; Jeon, S.-J.; Cho, B. R. Org. Lett. 2005, 7, 323-326.

(32) Nielsen, C. B.; Johnsen, M.; Arnbjerg, J.; Pittelkow, M.; McIlroy, S. P.; Ogilby, P. R.; Jorgensen, M. J. Org. Chem. 2005, 70, 7065-7079.

(33) Mongin, O.; Porrès, L.; Charlot, M.; Katan, C.; Blanchard-Desce, M. Chem. Eur. J. 2007, 13, 1481-1498.

(34) Chung, S.-J.; Kim, K.-S.; Lin, T.-C.; He, G. S.; Swiatkiewicz, J.; Prasad, P. N. J. Phys. Chem. B 1999, 103, 10741-10745.

(35) Cho, B. R.; Son, K. H.; Lee, S. H.; Song, Y.-S.; Lee, Y.-K.; Jeon, S.-J.; Choi, J. H.; Lee, H.; Cho, M. J. Am. Chem. Soc. 2001, 123, 10039-10045.

(36) Beljonne, D.; Wenseleers, W.; Zojer, E.; Shuai, Z.; Vogel, H.; Pond, S. J. K.; Perry, J. W.; Marder, S. R.; Brédas, J.-L. Adv. Funct. Mater. 2002, 12, 631-641.

(37) Abbotto, A.; Beverina, L.; Bozio, R.; Facchetti, A.; Ferrante, C.; Pagani, G. A.; Pedron, D.; Signorini, R. Chem. Commun. 2003, 2144-2145.

(38) Yoo, J.; Yang, S. K.; Jeong, M.-Y.; Ahn, H. C.; Jeon, S.-J.; Cho, B. R. Org. Lett. 2003, 5, 645-648.

(39) Le Droumaguet, C.; Mongin, O.; Werts, M. H. V.; Blanchard-Desce, M. Chem. Commun. 2005, 2802-2804.

(40) Katan, C.; Terenziani, F.; Mongin, O.; Werts, M. H. V.; Porrès, L.; Pons, T.; Mertz, J.; Tretiak, S.; Blanchard-Desce, M. J. Phys. Chem. A 2005, 109, 3024-3037.

(41) McIlroy, S. P.; Clo, E.; Nikolajsen, L.; Frederiksen, P. K.; Nielsen, C. B.; Mikkelsen, K. V.; Gothelf, K. V.; Ogilby, P. R. J. Org. Chem. 2005, 70, 1134-1146.

(42) Terenziani, F.; Le Droumaguet, C.; Katan, C.; Mongin, O.; Blanchard-Desce, M. ChemPhysChem 2007, 8, 723-734.

(43) Drobizhev, M.; Karotki, A.; Dzenis, Y.; Rebane, A.; Suo, Z.; Spangler, C. W. J. Phys. Chem. B 2003, 107, 7540-7543.

(44) Varnavski, O.; Yan, X.; Mongin, O.; Blanchard-Desce, M.; Goodson, T., III $J$. Phys. Chem. C 2007, 111, 149-162.

(45) Bhaskar, A.; Guda, R.; Haley, M. M.; Goodson, T., III J. Am. Chem. Soc. 2006, $128,13972-13973$.

(46) Williams-Harry, M.; Bhaskar, A.; Ramakrishna, G.; Goodson, T., III; Imamura, M.; Mawatari, A.; Nakao, K.; Enozawa, H.; Nishinaga, T.; Iyoda, M. J. Am. Chem. Soc. 2008, 130, 3252-3253.

(47) Ogawa, K.; Ohashi, A.; Kobuke, Y.; Kamada, K.; Ohta, K. J. Am. Chem. Soc. 2003, 125, 13356-13357.

(48) Drobizhev, M.; Stepanenko, Y.; Dzenis, Y.; Karotki, A.; Rebane, A.; Taylor, P. N.; Anderson, H. L. J. Am. Chem. Soc. 2004, 126, 15352-15353.

(49) Ahn, T. K.; Kim, K. S.; Kim, D. Y.; Noh, S. B.; Aratani, N.; Ikeda, C.; Osuka, A.; Kim, D. J. Am. Chem. Soc. 2006, 128, 1700-1704. 
(50) Rath, H.; Sankar, J.; PrabhuRaja, V.; Chandrashekar, T. K.; Nag, A.; Goswami, D. J. Am. Chem. Soc. 2005, 127, 11608-11609.

(51) Mongin, O.; Krishna, T. R.; Werts, M. H. V.; Caminade, A.-M.; Majoral, J.-P.; Blanchard-Desce, M. Chem. Commun. 2006, 915-917.

(52) Pu, S.-C.; Yang, M.-J.; Hsu, C.-C.; Lai, C.-W.; Hsieh, C.-C.; Lin, S. H.; Cheng, Y.-M.; Chou, P.-T. Small 2006, 2, 1308-1313.

(53) Kim, S.; Ohulchanskyy, T. Y.; Pudavar, H. E.; Pandey, R. K.; Prasad, P. N. J. Am. Chem. Soc. 2007, 129, 2669-2675.

(54) Lebret, V.; Raehm, L.; Durand, J.-O.; Smaihi, M.; Gerardin, C.; Nerambourg, N.; Werts, M. H. V.; Blanchard-Desce, M. Chem. Mater. 2008, 20, 2174-2183.

(55) Terenziani, F.; Painelli, A.; Katan, C.; Charlot, M.; Blanchard-Desce, M. J. Am. Chem. Soc. 2006, 128, 15742-15755.

(56) Stahl, R.; Lambert, C.; Kaiser, C.; Wortmann, R.; Jakober, R. Chem. Eur. J. 2006, 12, 2358-2370.

(57) Terenziani, F.; Sissa, C.; Painelli, A. J. Phys. Chem. B 2008, 112, 5079-5087.

(58) Varnavski, O. P.; Ostrowski, J. C.; Sukhomlinova, L.; Twieg, R. J.; Bazan, G. C.; Goodson, T., III J. Am. Chem. Soc. 2002, 124, 1736-1743.

16849.

(59) Liu, L. A.; Peteanu, L. A.; Yaron, D. J. J. Phys. Chem. B 2004, 108, 16841-

(60) Bangal, P. R.; Lam, D. M. K.; Peteanu, L. A.; VanderAuweraer, M. J. Phys. Chem. B 2004, 108, 16834-16840.

(61) Scherer, D.; Dörfler, R.; Feldner, A.; Vogtmann, T.; Schwoerer, M.; Lawrentz, U.; Grahn, W.; Lambert, C. Chem. Phys. 2002, 279, 179-207.

(62) Fu, J.; Przhonska, O. V.; Padilha, L. A.; Hagan, D. J.; Van Stryland, E. W.; Belfield, K. D.; Bondar, M. V.; Slominsky, Y. L.; Kachkovski, A. D. Chem. Phys. 2006, 321, 257-268.

(63) Charlot, M.; Izard, N.; Mongin, O.; Riehl, D.; Blanchard-Desce, M. Chem. Phys. Lett. 2006, 417, 297-302.

(64) Käpplinger, C.; Beckert, R. Synthesis 2002, 1843-1850.

(65) Mallegol, T.; Gmouh, S.; Meziane, M. A. A.; Blanchard-Desce, M.; Mongin, O. Synthesis 2005, 1771-1774.

(66) Malachesky, P. A. Anal. Chem. 1969, 41, 1493-1494.

(67) Barzoukas, M.; Blanchard-Desce, M. J. Chem. Phys. 2000, 113, 3951-3959.

(68) The oxidation potential is also related to exchange interactions in the radical cation species which are expected to be similar in the whole series.

(69) Li, Z.; Cheng, J. P. J. Org. Chem. 2003, 68, 7350-7360.

(70) Amthor, S.; Lambert, C.; Dummler, S.; Fischer, I.; Schelter, J. J. Phys. Chem. A 2006, 110, 5204-5214.

(71) Brunel, J.; Mongin, O.; Jutand, A.; Ledoux, I.; Zyss, J.; Blanchard-Desce, M. Chem. Mater. 2003, 15, 4139-4148.

(72) Lippert, E. Z. Naturforsch. A 1955, 10, 541-545.

(73) Mataga, N.; Kaifu, Y.; Koizumi, M. Bull. Chem. Soc. Jpn. 1955, 28, 690-691.

(74) Drobizhev, M.; Meng, F.; Rebane, A.; Stepanenko, Y.; Nickel, E.; Spangler, C. W. J. Phys. Chem. B 2006, 110, 9802-9814.

(75) Lakowicz, J. R. Principles of Fluorescence Spectroscopy, 3rd edition; Springer: New York, 2006.

(76) Liptay, W. In Excited States; Lim, E. C., Ed.; Academic Press: New York, 1974, p 129-229.

(77) Alain, V.; Rédoglia, S.; Blanchard-Desce, M.; Lebus, S.; Lukaszuk, K.; Wortmann, R.; Gubler, U.; Bosshard, C.; Günter, P. Chem. Phys. 1999, 245, 51-71. 
(78) Blanchard-Desce, M.; Wortmann, R.; Lebus, S.; Lehn, J.-M.; Kraemer, P. Chem. Phys. Lett. 1995, 243, 526-532.

(79) Blanchard-Desce, M.; Alain, V.; Midrier, L.; Wortmann, R.; Lebus, S.; Glania, C.; Kraemer, P.; Fort, A.; Muller, J.; Barzoukas, M. J. Photochem. Photobiol., A 1997, 105, 115-121.

(80) Rumi, M.; Ehrlich, J. E.; Heikal, A. A.; Perry, J. W.; Barlow, S.; Hu, Z.-Y.; McCord-Maughon, D.; Parker, T. C.; Röckel, H.; Thayumanavan, S.; Marder, S. R.; Beljonne, D.; Brédas, J.-L. J. Am. Chem. Soc. 2000, 122, 9500-9510.

(81) Katan, C.; Tretiak, S.; Werts, M. H. V.; Bain, A. J.; Marsh, R. J.; Leonczek, N.; Nicolaou, N.; Badaeva, E.; Mongin, O.; Blanchard-Desce, M. J. Phys. Chem. B 2007, 111, 9468-9483.

(82) Demas, J. N.; Crosby, G. A. J. Phys. Chem. 1971, 75, 991-1024.

(83) Eaton, D. F. Pure Appl. Chem. 1988, 60, 1107-1114.

(84) Xu, C.; Webb, W. W. J. Opt. Soc. Am. B 1996, 13, 481-491.

(85) Albota, M. A.; Xu, C.; Webb, W. W. Appl. Opt. 1998, 37, 7352-7356.

(86) Werts, M. H. V.; Nerambourg, N.; Pélégry, D.; Le Grand, Y.; BlanchardDesce, M. Photochem. Photobiol. Sci. 2005, 4, 531-538.

(87) Barzoukas, M.; Runser, C.; Fort, A.; Blanchard-Desce, M. Chem. Phys. Lett. 1996, 257, 531 .

(88) Blanchard-Desce, M.; Barzoukas, M. J. Opt. Soc. Am. B 1998, 15, 302-307.

(89) Painelli, A. Chem. Phys. Lett. 1998, 285, 352-358.

(90) Thompson, W. H.; Blanchard-Desce, M.; Hynes, J. T. J. Phys. Chem. A 1998, $102,7712-7722$.

(91) Barzoukas, M.; Blanchard-Desce, M. J. Chem. Phys. 2000, 112, 2036-2044.

(92) Painelli, A.; Del Freo, L.; Terenziani, F. Chem. Phys. Lett. 2001, 346, 470-478.

(93) Hahn, S.; Kim, D.; Cho, M. J. Phys. Chem. B 1999, 103, 8221-8229.

(94) Lee, W.-H.; Lee, H.; Kim, J.-A.; Choi, J.-H.; Cho, M.; Jeon, S.-J.; Cho, B. R. J. Am. Chem. Soc. 2001, 123, 10658-10667.

(95) Drobizhev, M.; Makarov, N. S.; Stepanenko, Y.; Rebane, A. J. Chem. Phys. 2006, 124, 224701.

(96) Painelli, A. Chem. Phys. 1999, 245, 185-197.

(97) Gaussian 03, Revision D.02, Frisch, M. J.; Trucks, G. W.; Schlegel, H. B.; Scuseria, G. E.; Robb, M. A.; Cheeseman, J. R.; Montgomery, Jr., J. A.; Vreven, T.; Kudin, K. N.; Burant, J. C.; Millam, J. M.; Iyengar, S. S.; Tomasi, J.; Barone, V.; Mennucci, B.; Cossi, M.; Scalmani, G.; Rega, N.; Petersson, G. A.; Nakatsuji, H.; Hada, M.; Ehara, M.; Toyota, K.; Fukuda, R.; Hasegawa, J.; Ishida, M.; Nakajima, T.; Honda, Y.; Kitao, O.; Nakai, H.; Klene, M.; Li, X.; Knox, J. E.; Hratchian, H. P.; Cross, J. B.; Bakken, V.; Adamo, C.; Jaramillo, J.; Gomperts, R.; Stratmann, R. E.; Yazyev, O.; Austin, A. J.; Cammi, R.; Pomelli, C.; Ochterski, J. W.; Ayala, P. Y.; Morokuma, K.; Voth, G. A.; Salvador, P.; Dannenberg, J. J.; Zakrzewski, V. G.; Dapprich, S.; Daniels, A. D.; Strain, M. C.; Farkas, O.; Malick, D. K.; Rabuck, A. D.; Raghavachari, K.; Foresman, J. B.; Ortiz, J. V.; Cui, Q.; Baboul, A. G.; Clifford, S.; Cioslowski, J.; Stefanov, B. B.; Liu, G.; Liashenko, A.; Piskorz, P.; Komaromi, I.; Martin, R. L.; Fox, D. J.; Keith, T.; Al-Laham, M. A.; Peng, C. Y.; Nanayakkara, A.; Challacombe, M.; Gill, P. M. W.; Johnson, B.; Chen, W.; Wong, M. W.; Gonzalez, C.; and Pople, J. A.; Gaussian, Inc., Wallingford CT, 2004.

(98) Casida, M. E.; Jamorski, C.; Casida, K. C.; Salahub, D. R. J. Chem. Phys. 1998, 108, 4439-4449.

(99) Furche, F.; Ahlrichs, R. J. Chem. Phys. 2002, 117, 7433-7447.

(100) Onida, G.; Reining, L.; Rubio, A. Rev. Mod. Phys. 2002, 74, 601-659.

(101) Tretiak, S.; Chernyak, V. J. Chem. Phys. 2003, 119, 8809-8823. 
J. Phys. Chem. B, 2010, 114 (9), pp 3152-3169 ; DOI: 10.1021/jp911445m

(102) Garito, A. F.; Heflin, J. R.; Wong, K. Y.; Zamani-Khamiri, O. In Organic Materials for Non-linear Optics; Hann, R. A., Bloor, D., Eds.; Royal Society of Chemistry: London, 1989, pp 16-27.

(103) Ahlrichs, R.; Bär, M.; Häser, M.; Horn, H.; Kölmel, C. Chem. Phys. Lett. 1989, $162,165-169$.

(104) Martin, R. L. J. Chem. Phys. 2003, 118, 4775-4777.

(105) Kokalj, A. J. Mol. Graphics Modell. 2000, 17, 176-179. 
J. Phys. Chem. B, 2010, 114 (9), pp 3152-3169 ; DOI: 10.1021/jp911445m

Table 1. Electrochemical properties from cyclic voltammetry of quadrupolar-type compounds Q1-Q3 in THF/CH $\mathrm{CH}_{3} \mathrm{CN}$ and octupolar-type compounds $\mathbf{0 1 - 0 3}$ in $\mathrm{CH}_{3} \mathrm{CN}$

\begin{tabular}{cccccccc}
\hline & $\begin{array}{c}\mathrm{E}_{\mathrm{p}}^{\text {ox } a} \\
{[\mathrm{~V}]}\end{array}$ & $\begin{array}{c}\mathrm{E}^{0} \\
{[\mathrm{~V}]}\end{array}$ & $\begin{array}{c}\left(\mathrm{E}_{\mathrm{p}}-\mathrm{E}_{\mathrm{p} / 2}\right) \\
{[\mathrm{V}]}\end{array}$ & $\mathrm{i}_{\mathrm{c}} / \mathrm{i}_{\mathrm{a}}{ }^{b}$ & $\mathrm{n}^{\text {ox } c}$ & $\begin{array}{c}\mathrm{E}_{\mathrm{p}}^{\text {red } a} \\
{[\mathrm{~V}]}\end{array}$ & $\mathrm{n}^{\text {red } c}$ \\
\hline Q1 & $0.56_{5}$ & $0.52_{3}$ & 59 & 0.9 & 0.9 & - & - \\
Q2 & $0.55_{5}$ & $0.51_{3}$ & 64 & 0.9 & 0.9 & - & - \\
Q3 & $0.52_{3}$ & $0.49_{6}$ & 62 & 1.0 & 0.9 & - & - \\
O1 & $0.45_{8}$ & $0.44_{5}$ & 62 & 0.9 & 1.2 & $-2.28_{5}$ & 1.0 \\
O2 & $0.47_{8}$ & $0.45_{9}$ & 65 & 0.8 & 1.0 & $-2.10_{5}$ & 0.95 \\
O3 & $0.52_{0}$ & $0.46_{4}$ & 72 & 0.9 & 1.1 & $-2.54_{5}$ & 1.1 \\
\hline
\end{tabular}

${ }^{a}$ Potentials vs. Ag/0.1 $\mathrm{M} \mathrm{AgNO}_{3} .{ }^{b}$ Ratio of cathodic to anodic peak currents. ${ }^{c}$ Absolute electron number value, see ${ }^{66}$. 
J. Phys. Chem. B, 2010, 114 (9), pp 3152-3169 ; DOI: 10.1021/jp911445m

Table 2. Photophysical properties of chromophores Q1-Q3 and O1-O3 in toluene

\begin{tabular}{|c|c|c|c|c|c|c|c|c|c|c|c|}
\hline & $\begin{array}{c}\lambda_{\max 2}^{a}{ }^{a b s} \\
{[\mathrm{~nm}]}\end{array}$ & $\begin{array}{c}\varepsilon_{\max 2}^{b} \\
{\left[\mathrm{~cm}^{-1} \mathrm{M}^{-1}\right]}\end{array}$ & $\begin{array}{c}\lambda_{\max 1}^{a}{ }^{a b s} \\
{[\mathrm{~nm}]}\end{array}$ & $\begin{array}{c}\varepsilon_{\max 1}^{b} \\
{\left[\mathrm{~cm}^{-1} \mathrm{M}^{-1}\right]}\end{array}$ & $\begin{array}{c}\lambda_{\max }^{\mathrm{em}}{ }^{\mathrm{em}} \\
{[\mathrm{nm}]}\end{array}$ & $\begin{array}{l}\mathrm{E}_{0-0}{ }^{d} \\
{[\mathrm{eV}]}\end{array}$ & $\begin{array}{c}\text { Stokes shift } \\
e \\
{\left[\mathrm{~cm}^{-1}\right]}\end{array}$ & $\Phi^{f}$ & $\begin{array}{c}\tau^{g} \\
{[\mathrm{~ns}]}\end{array}$ & $\begin{array}{c}k_{r}^{h} \\
{\left[10^{9} \mathrm{~s}^{-1}\right]}\end{array}$ & $\begin{array}{c}k_{n r}{ }^{h} \\
{\left[10^{9} \mathrm{~s}^{-1}\right]}\end{array}$ \\
\hline Q1 & 1 & 1 & 387 & 83000 & 421 & 3.00 & 2100 & 0.80 & 0.74 & 1.08 & 0.27 \\
\hline Q2 & / & I & 377 & 65900 & 417 & 3.04 & 2500 & 0.69 & 0.77 & 0.90 & 0.40 \\
\hline Q3 & 1 & 1 & 366 & 65800 & 400 & 3.16 & 2300 & 0.74 & 0.67 & 1.10 & 0.39 \\
\hline O1 & 308 & 43500 & 430 & 80800 & 494 & 2.65 & 3000 & 0.71 & 1.85 & 0.38 & 0.16 \\
\hline $\mathbf{O 2}$ & 306 & 37200 & 425 & 68400 & 498 & 2.65 & 3500 & 0.77 & 1.75 & 0.44 & 0.13 \\
\hline O3 & 304 & - & 415 & - & 469 & 2.67 & 2750 & 0.70 & 1.94 & 0.36 & 0.15 \\
\hline
\end{tabular}

${ }^{a}$ Experimental absorption maxima. ${ }^{b}$ Molar extinction coefficients. ${ }^{c}$ Experimental emission maximum. ${ }^{d}$ Electronic gap (0-0 transition energy) estimated from the intersection of normalized absorption and emission spectra in toluene. ${ }^{e}$ Stokes shift $=\left(1 / \lambda_{\mathrm{abs}}-1 / \lambda_{\mathrm{em}}\right) .{ }^{f}$ Fluorescence quantum yield determined relative to fluorescein in $0.1 \mathrm{~N} \mathrm{NaOH} .{ }^{g}$ Experimental fluorescence lifetime. ${ }^{h}$ Radiative $\left(k_{r}\right)$ and non-radiative $\left(k_{\mathrm{nr}}\right)$ decay rates derived from fluorescence quantum yield and lifetime values $\left(k_{r}=\Phi / \tau ; k_{n r}=(1-\Phi) / \tau\right)$. 
J. Phys. Chem. B, 2010, 114 (9), pp 3152-3169 ; DOI: 10.1021/jp911445m

Table 3. Calculated photophysical properties of chromophores Q1-Q3 and O1-O3 in vacuum

\begin{tabular}{|c|c|c|c|c|c|c|c|c|c|c|}
\hline & $\begin{array}{c}\lambda_{\max 2}^{a}{ }^{a b s} \\
{[\mathrm{~nm}]}\end{array}$ & $\begin{array}{c}\alpha_{\max 2}^{b} \\
{\left[\mathrm{D}^{2} \mathrm{eV}^{-1}\right]}\end{array}$ & $\begin{array}{c}\lambda_{\max 1}^{a b s}{ }^{a b} \\
{[\mathrm{~nm}]}\end{array}$ & $\begin{array}{c}\alpha_{\max 1}^{b} \\
{\left[\mathrm{D}^{2} \mathrm{eV}^{-1}\right]}\end{array}$ & $\begin{array}{c}\lambda_{\max }^{\mathrm{em}}{ }^{c} \\
{[\mathrm{~nm}]}\end{array}$ & $\begin{array}{c}\mu^{\mathrm{em}} \\
{[\mathrm{D}]}\end{array}$ & $\begin{array}{l}\mathrm{E}_{0-0}{ }^{d} \\
{[\mathrm{eV}]}\end{array}$ & $\begin{array}{l}\text { Stokes } \\
\text { shift } \\
{\left[\mathrm{cm}^{-1}\right]}\end{array}$ & $\begin{array}{c}\tau_{0}^{f} \\
{[\mathrm{~ns}]}\end{array}$ & $\begin{array}{c}\mathrm{k}_{\mathrm{r}}{ }^{g} \\
{\left[10^{9} \mathrm{~s}^{-1}\right]}\end{array}$ \\
\hline Q1 & 290 & 280 & 390 & 1120 & 447 & 15.8 & 3.47 & 3300 & 1.14 & 0.88 \\
\hline Q2 & 282 & 240 & 383 & 1050 & 441 & 15.3 & 3.50 & 3400 & 1.16 & 0.86 \\
\hline Q3 & 277 & 210 & 377 & 1030 & 433 & 15.0 & 3.58 & 3400 & 1.15 & 0.87 \\
\hline O1 & 316 & 780 & 451 & 1110 & 506 & 13.1 & 3.03 & 2400 & 2.43 & 0.41 \\
\hline $\mathbf{O 2}$ & 315 & 650 & 448 & 970 & 509 & 12.7 & 2.99 & 2700 & 2.60 & 0.38 \\
\hline $\mathbf{O 3}$ & 307 & 750 & 430 & 1130 & 489 & 13.0 & 3.19 & 2800 & 2.20 & 0.45 \\
\hline
\end{tabular}

${ }^{a}$ Calculated absorption maxima. ${ }^{b}$ Imaginary part of the first order polarisability. ${ }^{c}$ Calculated emission maximum. ${ }^{d}$ HOMO-LUMO gap. ${ }^{e}$ Stokes shift $=\left(1 / \lambda_{\mathrm{abs}}-1 / \lambda_{\mathrm{em}}\right) .{ }^{f}$ Calculated radiative lifetime. ${ }^{g}$ Calculated radiative decay rate. 
J. Phys. Chem. B, 2010, 114 (9), pp 3152-3169 ; DOI: 10.1021/jp911445m

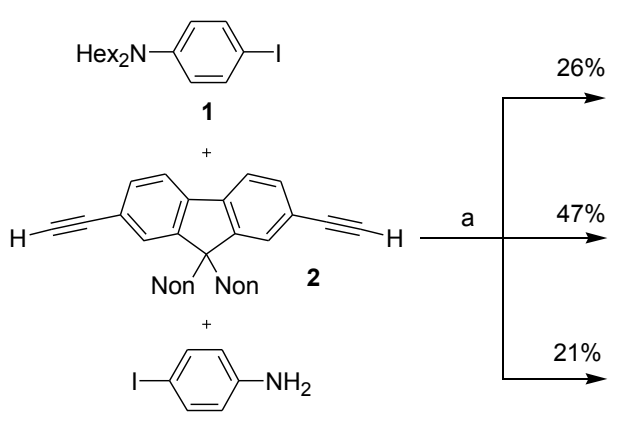

3
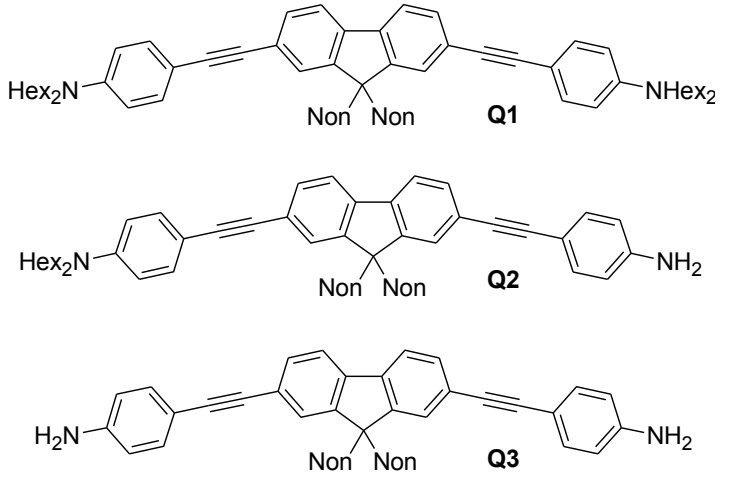

SCHEME 1: Synthesis of chromophores Q1-Q3 ${ }^{a}$

${ }^{a}$ Reagents and conditions: (a) 1 (1 equiv.), 2 (1 equiv.), 3 (1 equiv.), $\mathrm{Pd}\left(\mathrm{PPh}_{3}\right)_{2} \mathrm{Cl}_{2}, \mathrm{CuI}, \mathrm{Et}_{3} \mathrm{~N}$, $35^{\circ} \mathrm{C}$. Non $=n$-nonyl. 


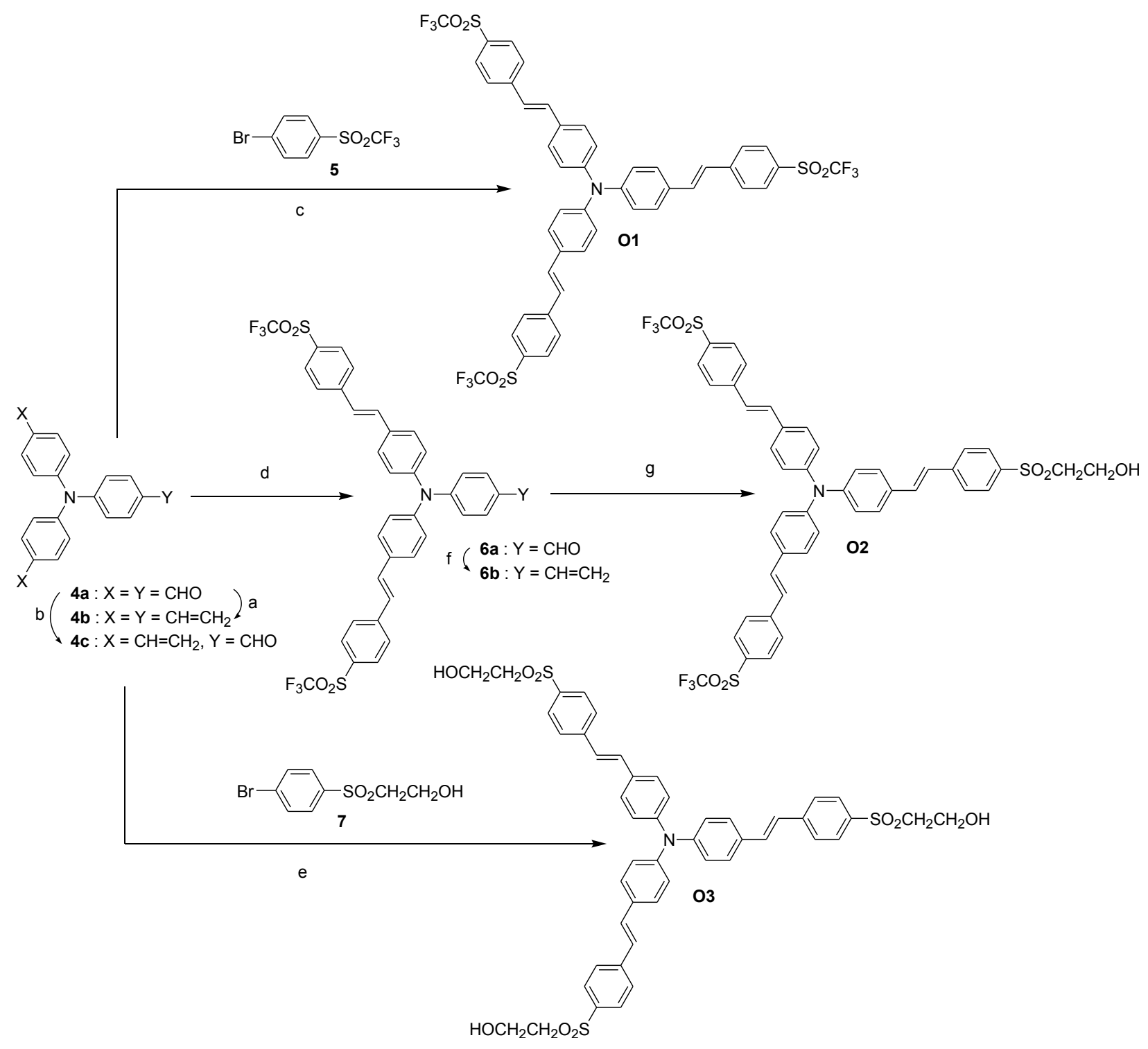

SCHEME 2: Synthesis of chromophores O1-O3 ${ }^{a}$

${ }^{a}$ Reagents and conditions: (a) methyltriphenylphosphonium iodide (excess), NaH, THF, $\mathrm{rt}$ (99\%); (b) methyltriphenylphosphonium iodide (1.5 equiv), NaH, THF, rt (31\%); (c) 4b (1 equiv), 5 (3.5 equiv), $\mathrm{Pd}(\mathrm{OAc})_{2},(o \text {-tol })_{3} \mathrm{P}, \mathrm{Et}_{3} \mathrm{~N}, \mathrm{DMF}, 100^{\circ} \mathrm{C}(75 \%)$; (d) $4 \mathbf{c}$ (1 equiv), 5 (2.3 equiv), $\mathrm{Pd}(\mathrm{OAc})_{2},(o \text {-tol })_{3} \mathrm{P}, \mathrm{Et}_{3} \mathrm{~N}, \mathrm{DMF}, 100^{\circ} \mathrm{C}(70 \%)$; (e) $4 \mathbf{b}$ (1 equiv), 7 (3.5 equiv), $\mathrm{Pd}(\mathrm{OAc})_{2},(o \text {-tol })_{3} \mathrm{P}, \mathrm{Et}_{3} \mathrm{~N}, \mathrm{DMF}, 100^{\circ} \mathrm{C}(42 \%)$; (f) methyltriphenylphosphonium iodide, $\mathrm{NaH}, \mathrm{THF}, \mathrm{rt}(54 \%)$; (g) $6 \mathbf{b}$ (1 equiv), 7 (1.3 equiv), $\mathrm{Pd}(\mathrm{OAc})_{2},(o \text {-tol })_{3} \mathrm{P}, \mathrm{Et}_{3} \mathrm{~N}, \mathrm{DMF}, 100^{\circ} \mathrm{C}$ $(53 \%)$. 
J. Phys. Chem. B, 2010, 114 (9), pp 3152-3169 ; DOI: 10.1021/jp911445m

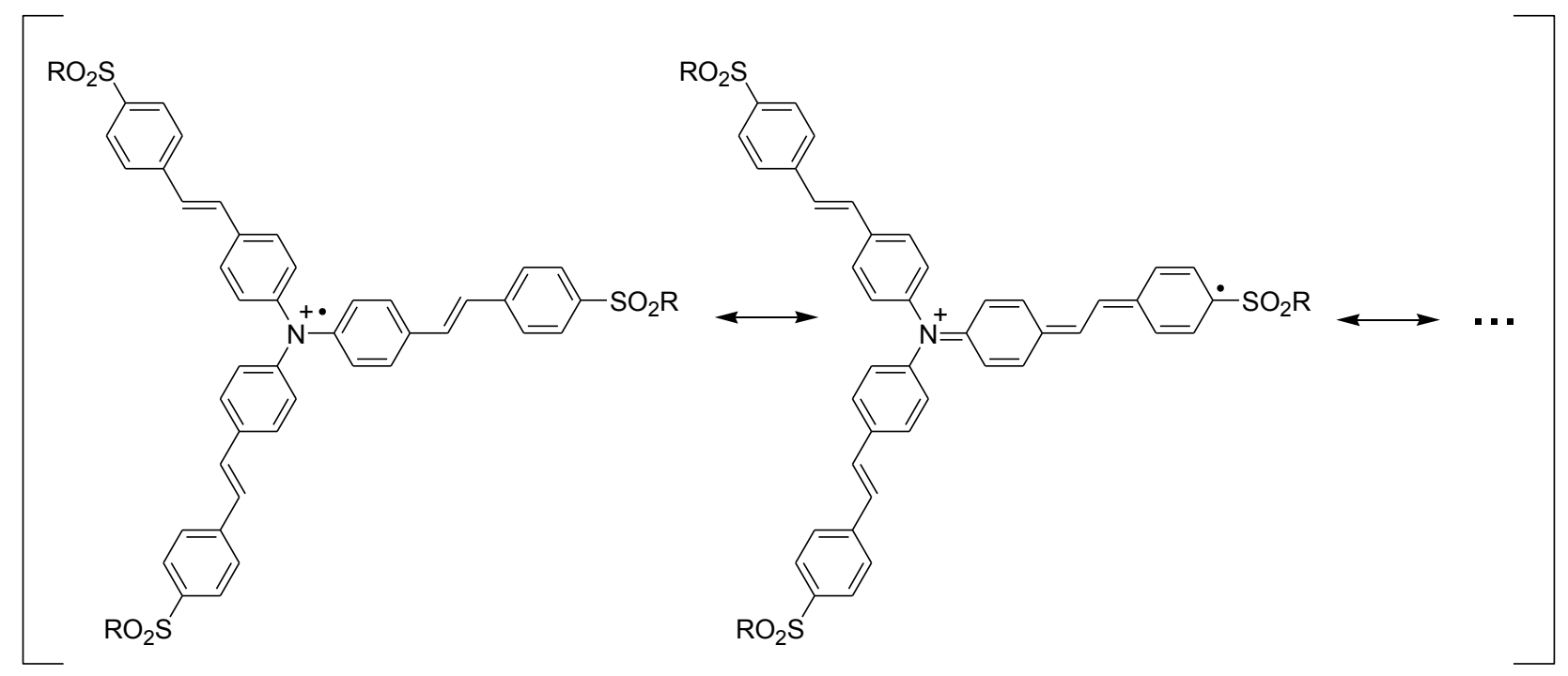

SCHEME 3: Stabilization of the radical cation of the octupolar derivatives. 


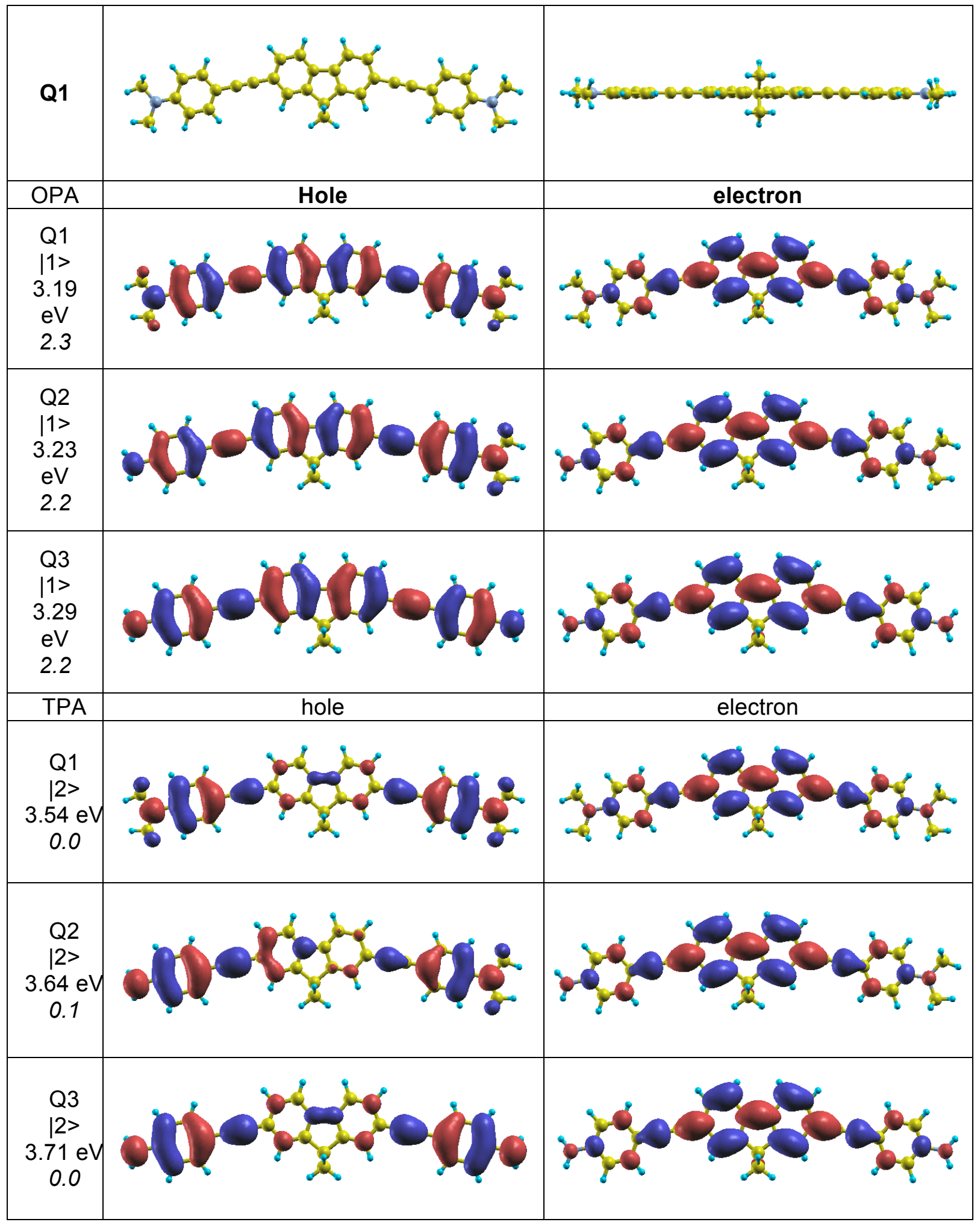

Figure 1. Optimized geometry of quadrupole $\mathbf{Q 1}$ and natural transition orbitals ${ }^{104}$ of the first two excited states for quadrupoles Q1-Q3. Left panels quote in sequence quadrupole label, excited state number, associated transition energy followed by oscillator strength in italic. For the sake of simplicity, the alkyl chains have been replaced by methyl groups. 
J. Phys. Chem. B, 2010, 114 (9), pp 3152-3169 ; DOI: 10.1021/jp911445m

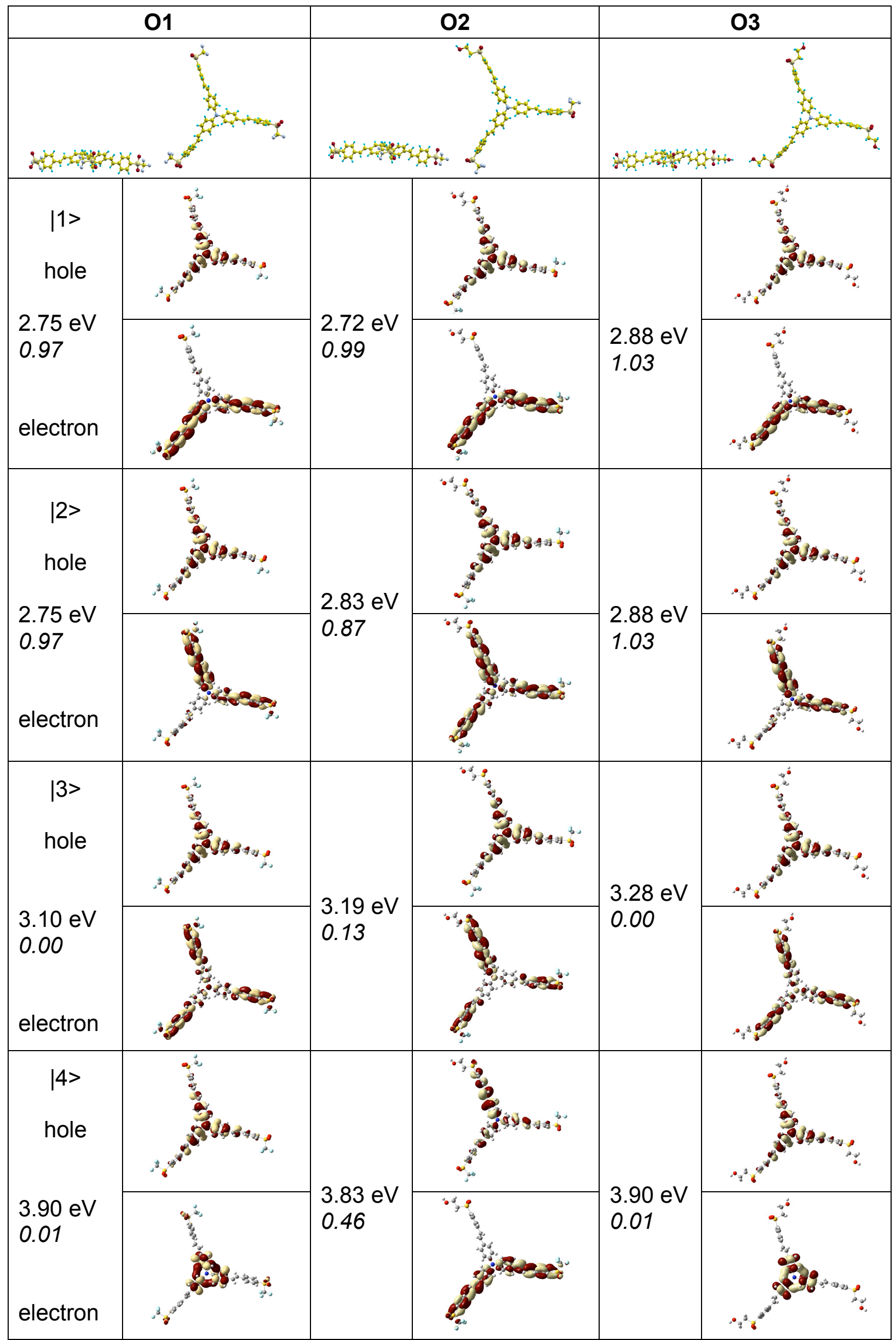


J. Phys. Chem. B, 2010, 114 (9), pp 3152-3169 ; DOI: 10.1021/jp911445m

Figure 2. Optimized geometries of octupoles $\mathrm{O} 1-\mathrm{O} 3$ and corresponding natural transition orbitals ${ }^{104}$ of the first four excited states of octupoles O1-O3. Excited state number are given in left panels, additional values quote in sequence associated transition energy followed by oscillator strength in italic. 


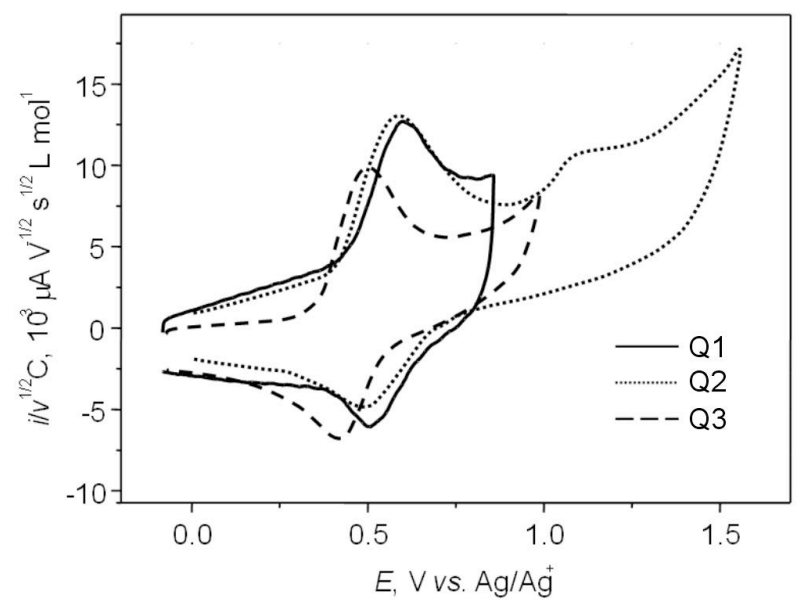

Figure 3. Normalized voltammograms of oxidation of compounds Q1-Q3 (respectively D- $\pi$-D, D- $\pi$-D' and $\left.D^{\prime}-\pi-D^{\prime}\right)$ at a gold disk electrode in $0.1 \mathrm{M} \mathrm{Bu}_{4} \mathrm{NPF}_{6}$ solutions in $\mathrm{THF} / \mathrm{CH}_{3} \mathrm{CN}$ (3-5:1 $\mathrm{v} / \mathrm{v})$. Scan rates for Q1-Q3, respectively: $v=0.1,2.5,2.5 \mathrm{~V} \mathrm{~s}^{-1}$.

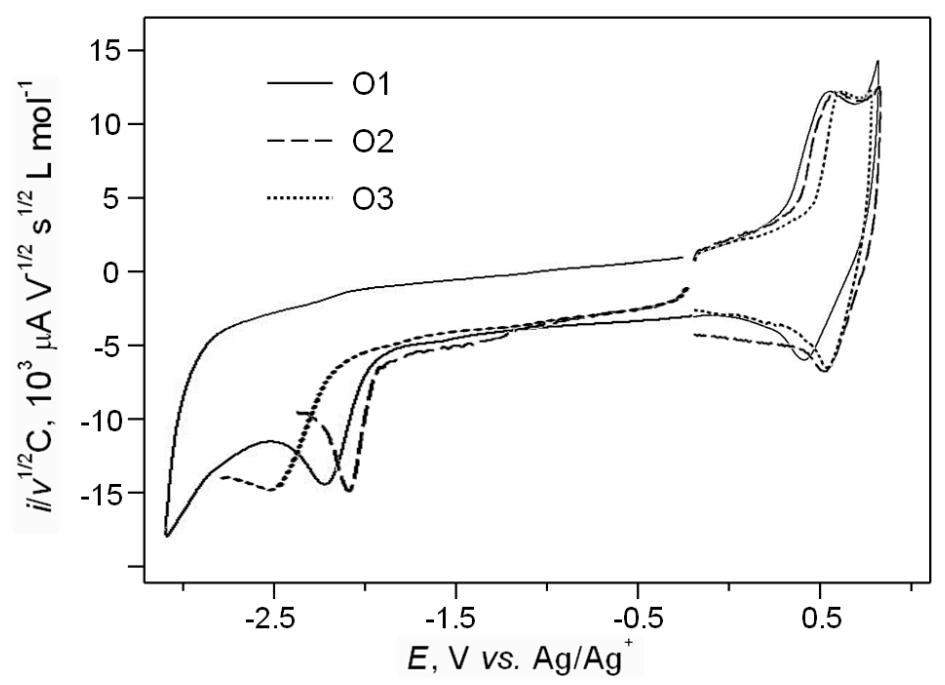

Figure 4. Normalized voltammograms of compounds $\mathbf{O 1 - O 3}$ at a GC disk electrode in $0.1 \mathrm{M}$ $\mathrm{Bu}_{4} \mathrm{NPF}_{6}$ solution in THF/CH $\mathrm{CH}_{3} \mathrm{CN}(5: 1 \mathrm{v} / \mathrm{v})$. Scan rate $v=1 \mathrm{~V} \mathrm{~s}^{-1}$. 
experimental
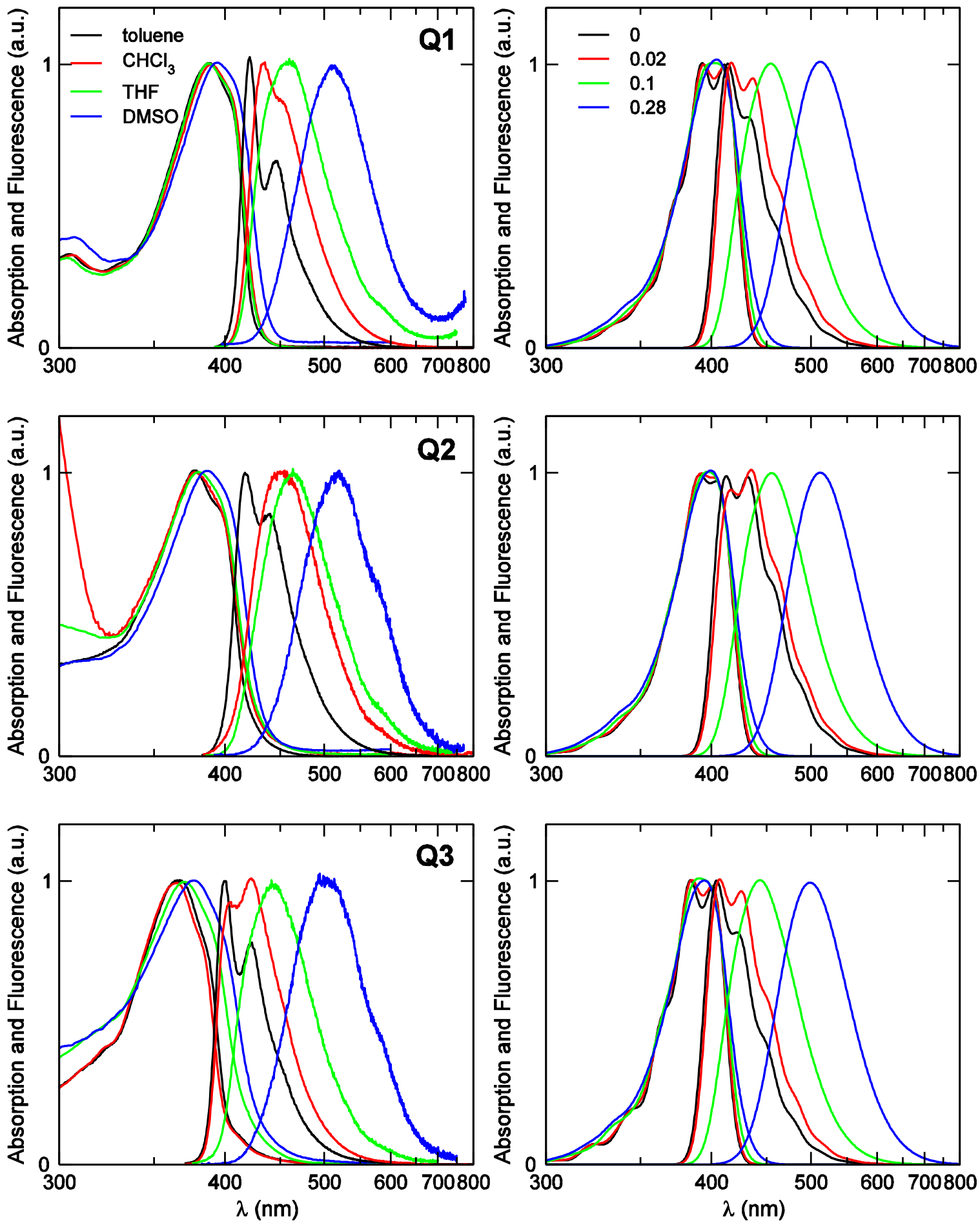

Figure 5. Left panels: experimental normalized absorption and emission spectra of Q1-Q3 in solvents of increasing polarity. Right panels: absorption and fluorescence spectra of chromophores Q1-Q3 calculated through a three-state model plus electron-phonon coupling plus solvation interaction. Parameters: $z=1.50 / 1.54 \mathrm{eV}$ for $\mathbf{Q 1} / \mathbf{Q 3}, z_{1 / 2}=1.50 / 1.54$ for $\mathbf{Q 2}, \sqrt{2} t=$ $0.60 \mathrm{eV}, \omega_{v}=0.16 \mathrm{eV}, \varepsilon_{v}=0.30 \mathrm{eV}, \mu_{0}=40 \mathrm{D}$. Gaussian lineshapes were used, with half-width at half maximum $\mathrm{HWHM}=0.08 \mathrm{eV}$. Values for $\varepsilon_{o r}$ in the legend $(\mathrm{eV})$. 
experimental
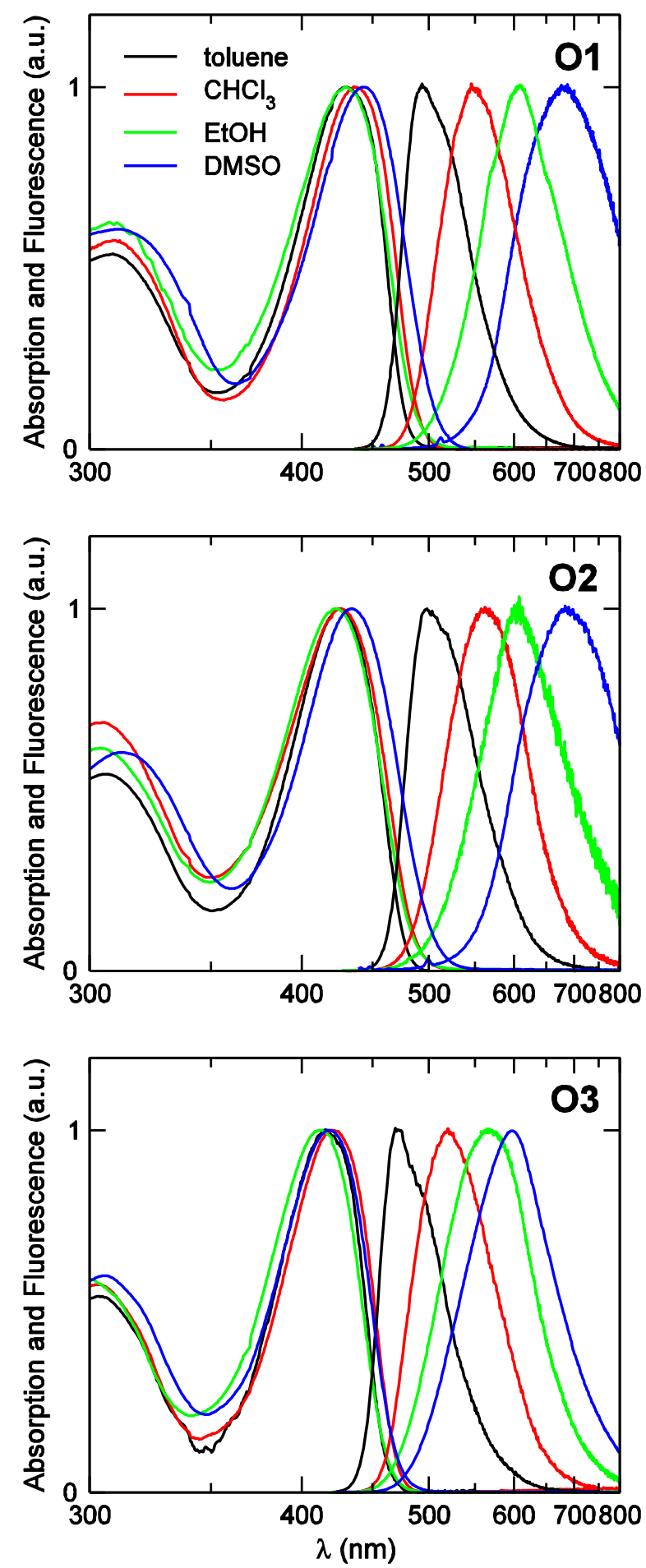

calculated
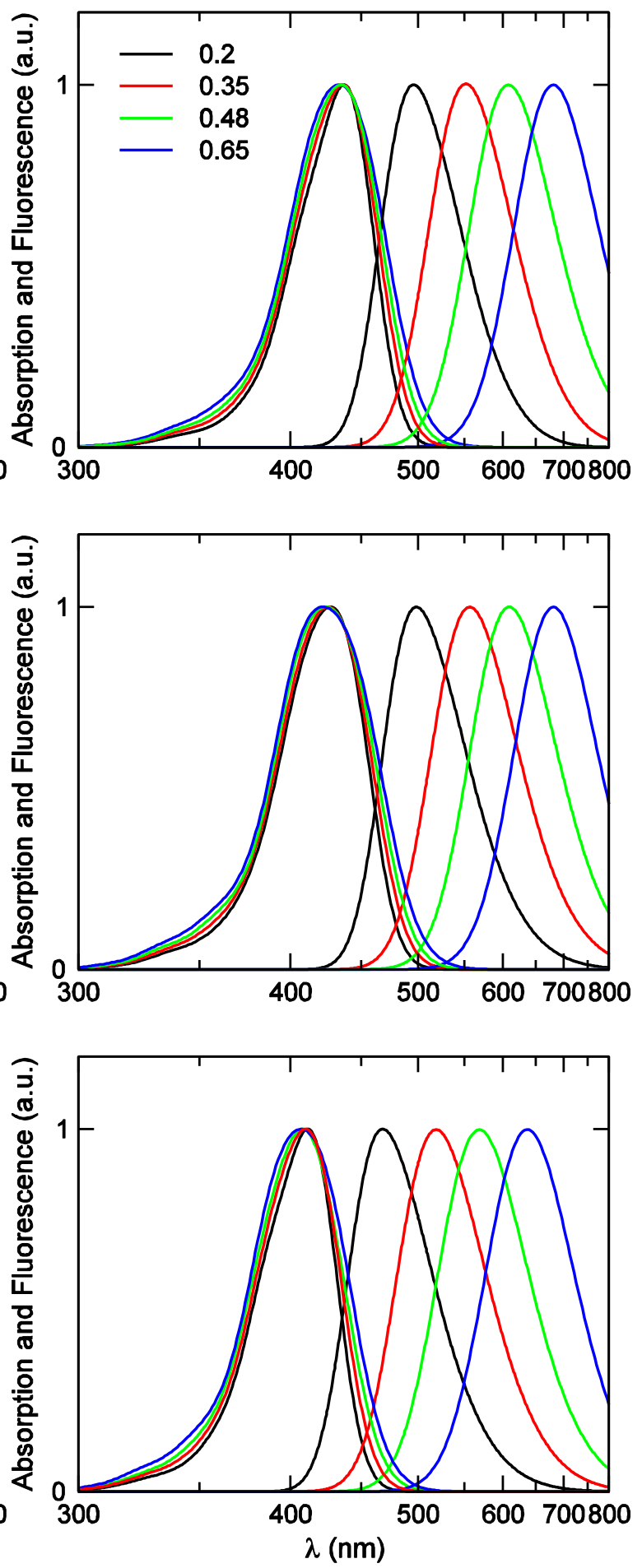

Figure 6. Left panels: experimental normalized absorption and emission spectra of 01-O3 in solvents of increasing polarity. Right panels: absorption and fluorescence spectra of chromophores O1-O3 calculated through a four-state model plus electron-phonon coupling plus solvation interaction. Parameters: $z=1.30 / 1.40 \mathrm{eV}$ for $\mathbf{0 1} / \mathbf{O 3}, z_{1 / 2}=1.30 / 1.40$ for $\mathbf{O 2}, \sqrt{2} t=$ $0.60 \mathrm{eV}, \omega_{v}=0.21 \mathrm{eV}, \varepsilon_{v}=0.19 \mathrm{eV}, \mu_{0}=33 \mathrm{D}$. Gaussian lineshapes were used, with half-width at half maximum $\mathrm{HWHM}=0.11 \mathrm{eV}$. Values for $\varepsilon_{o r}$ in the legend $(\mathrm{eV})$. 


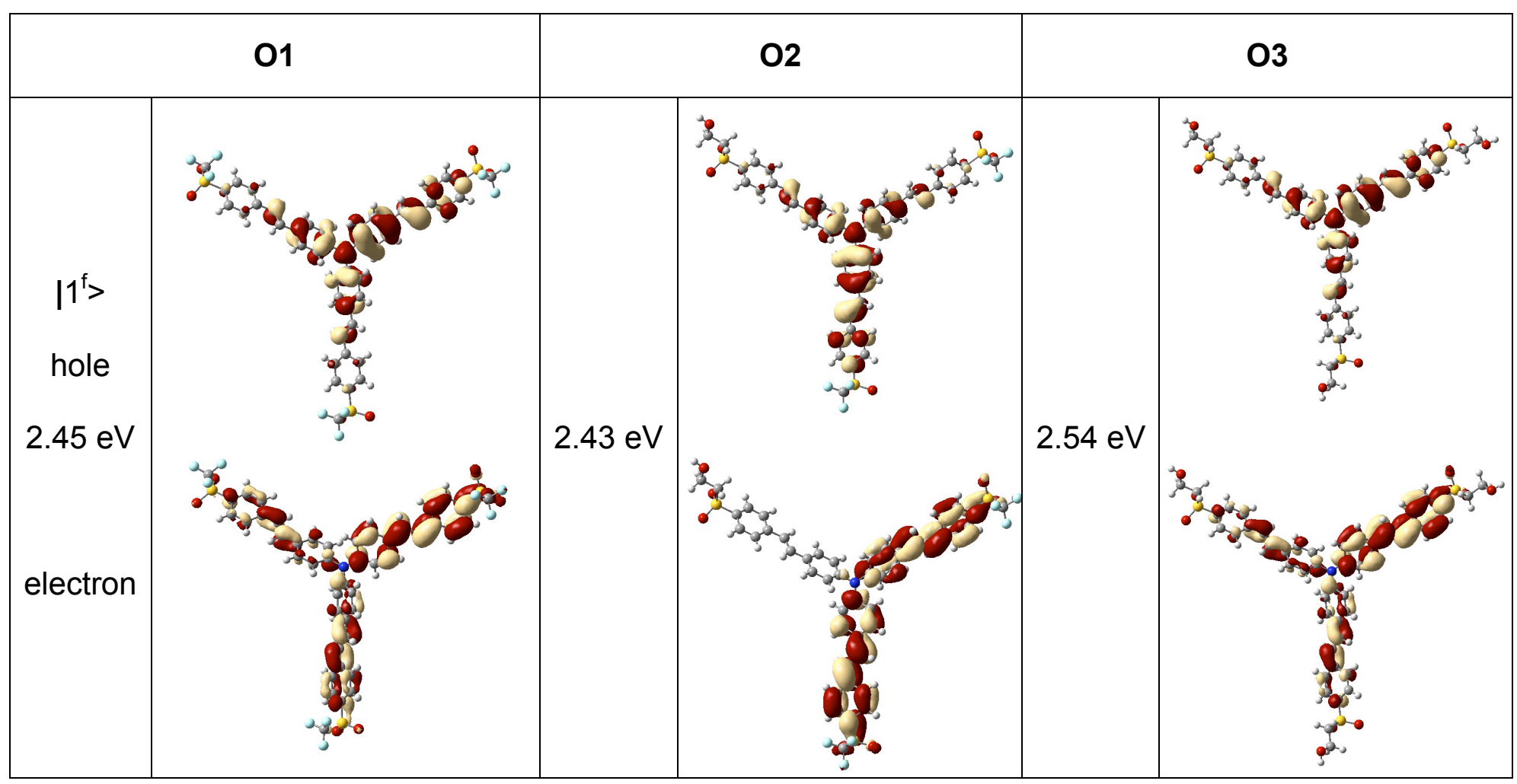

Figure 7. Natural transition orbitals ${ }^{104}$ relevant for emission (optimized excited state geometry) for octupolar derivatives O1-O3. 
J. Phys. Chem. B, 2010, 114 (9), pp 3152-3169 ; DOI: 10.1021/jp911445m
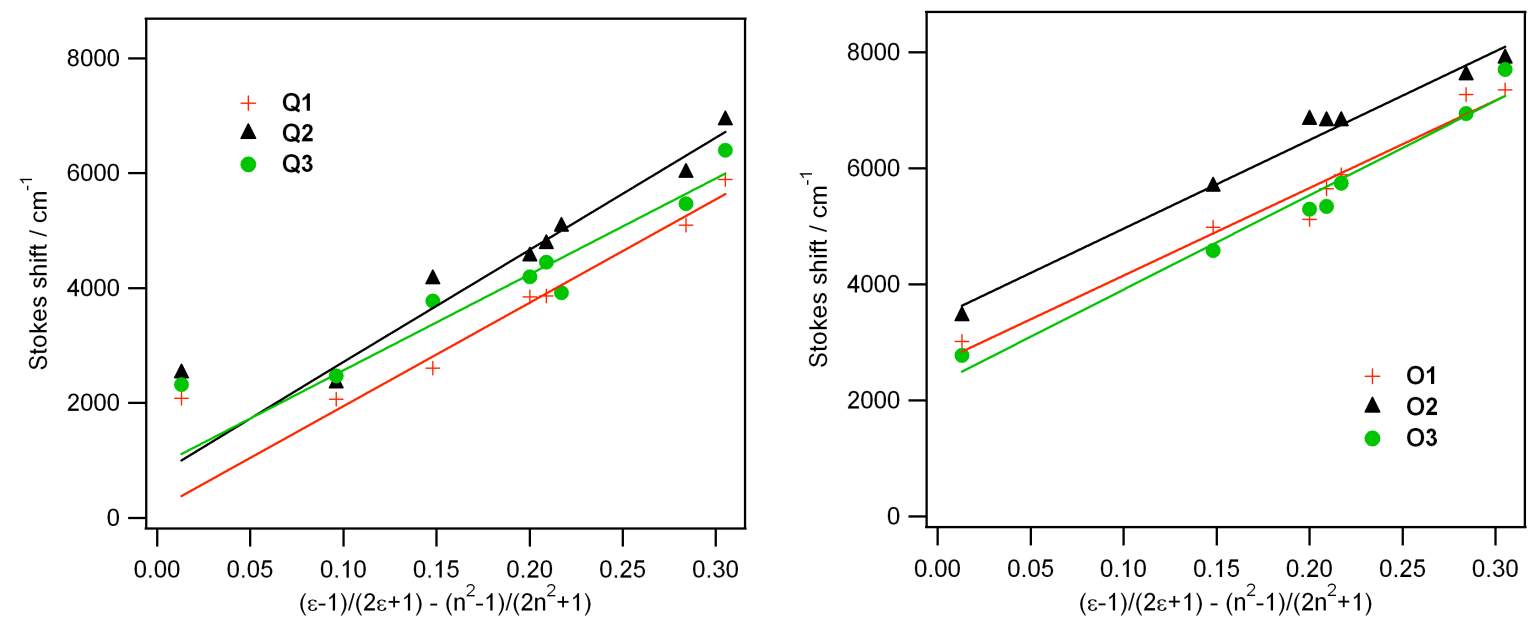

Figure 8. Lippert-Mataga correlations for chromophores Q1-Q3 (left) and O1-O3 (right). 
J. Phys. Chem. B, 2010, 114 (9), pp 3152-3169 ; DOI: 10.1021/jp911445m

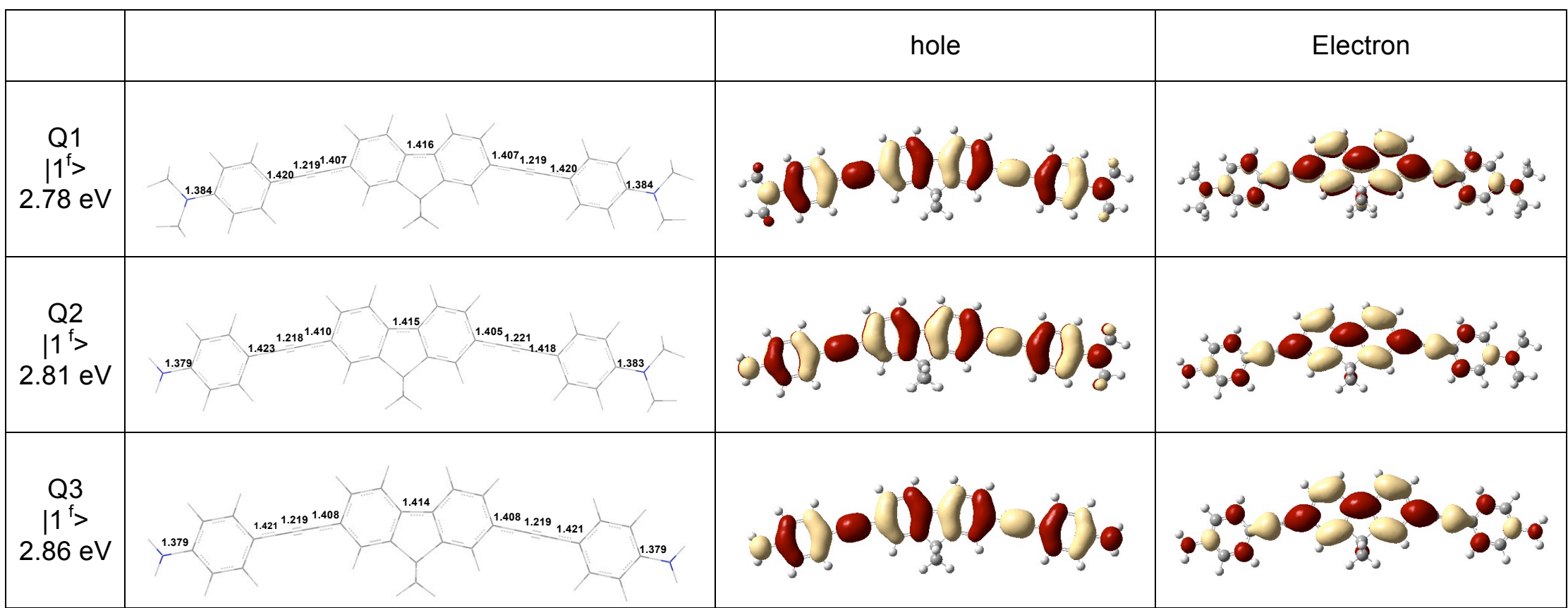

Figure 9. Optimized excited-state geometry and natural transition orbitals ${ }^{104}$ relevant for emission for quadrupoles Q1-Q3. For the sake of simplicity, the alkyl chains have been replaced by methyl groups. 

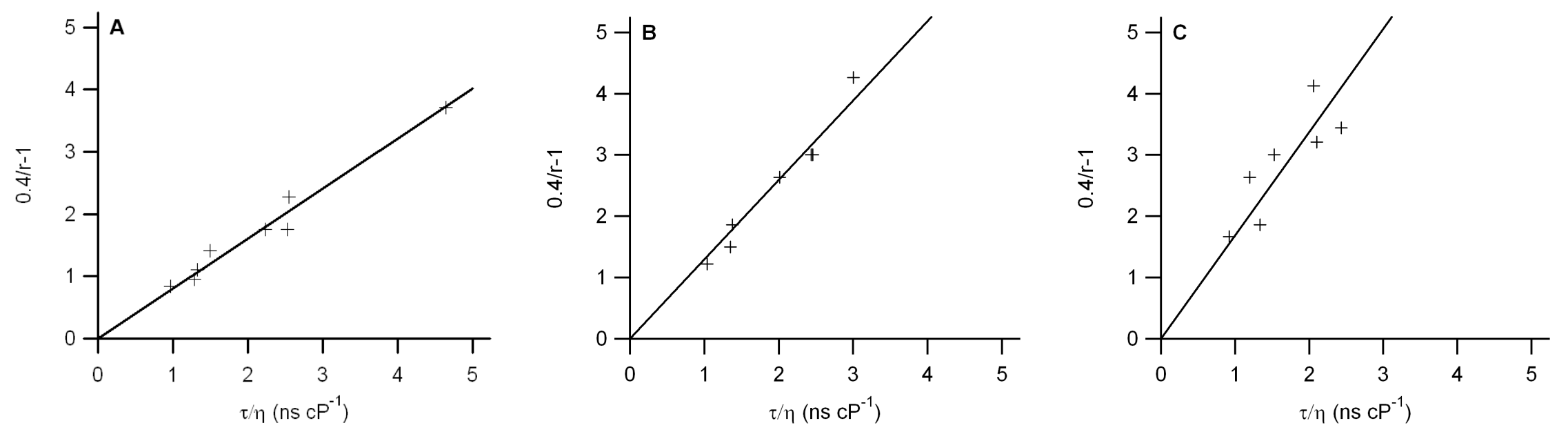

Figure 10. Perrin Plot for the anisotropy of fluorescence of chromophores Q1-Q3 measured in various solvents: Q1 (A), Q2(B), Q3 (C). 
J. Phys. Chem. B, 2010, 114 (9), pp 3152-3169 ; DOI: 10.1021/jp911445m

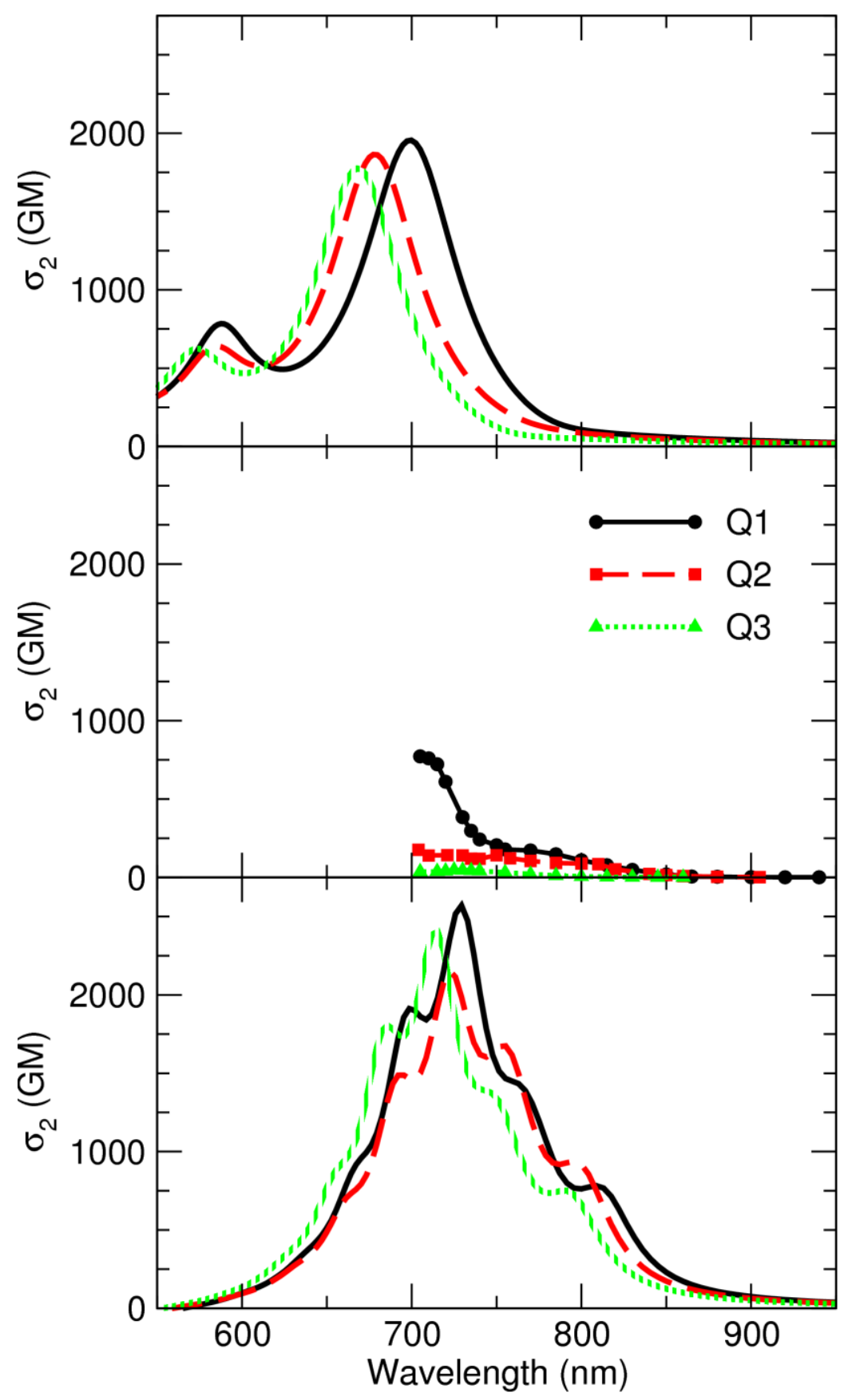

Figure 11. Two-Photon absorption cross-section for quadrupoles Q1-Q3: TD-B3LYP calculated data in vacuum (top), experimental data in toluene (middle) and spectra calculated through essential-state models for $\varepsilon_{o r}=0$ (bottom, see parameters in Figure 5). 
J. Phys. Chem. B, 2010, 114 (9), pp 3152-3169 ; DOI: 10.1021/jp911445m

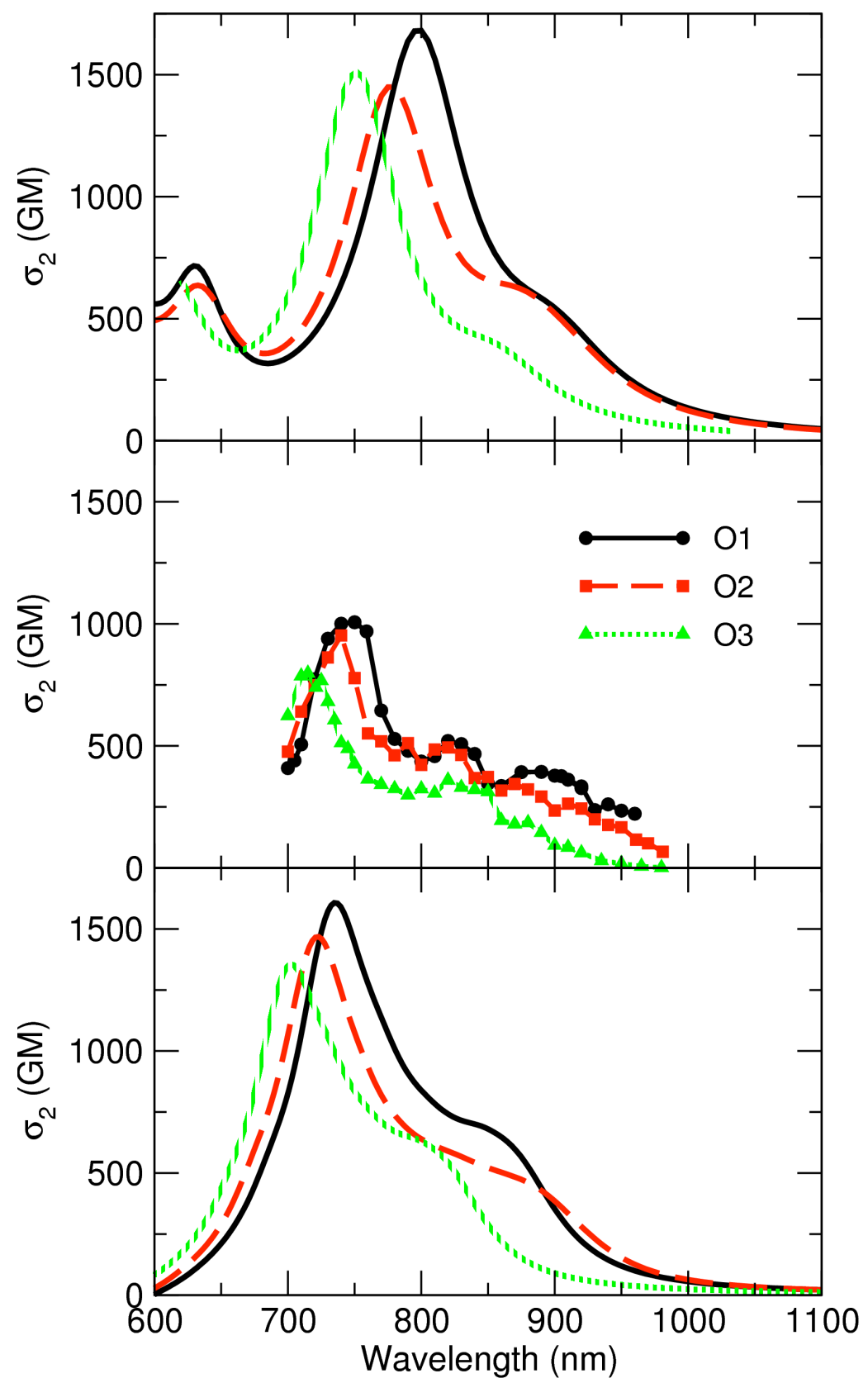

Figure 12. Two-Photon absorption cross-section of octupoles O1-O3: TD-B3LYP calculated data in vacuum (top), experimental data in ethanol (middle) and spectra calculated through essential-state models for $\varepsilon_{o r}=0.2$ (bottom, see parameters in Figure 6). 
J. Phys. Chem. B, 2010, 114 (9), pp 3152-3169 ; DOI: 10.1021/jp911445m

Table of Contents Graphic

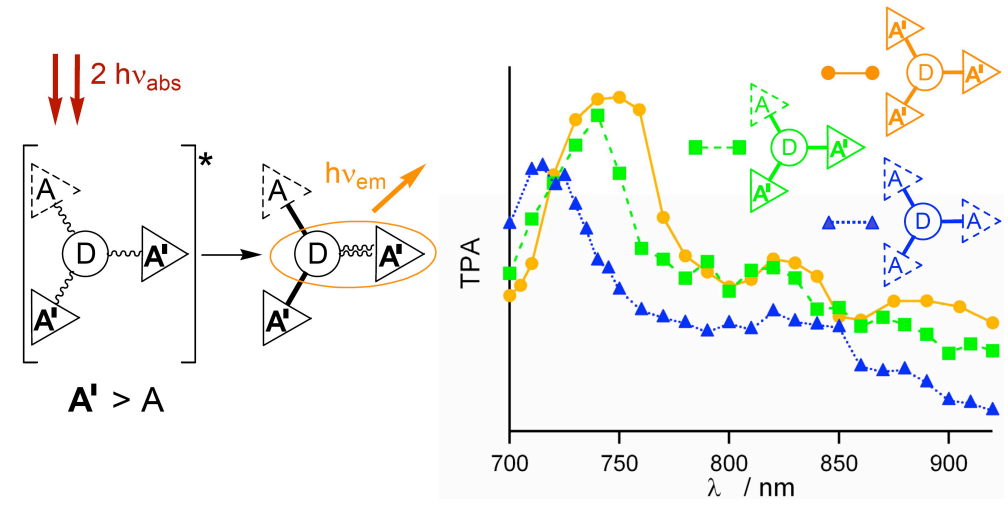

